\title{
CBX5/G9a/H3K9me-mediated gene repression is essential to fibroblast activation during lung fibrosis
}

\author{
Giovanni Ligresti, ${ }^{1}$ Nunzia Caporarello, ${ }^{1}$ Jeffrey A. Meridew, ${ }^{1}$ Dakota L. Jones, ${ }^{1}$ Qi Tan, ${ }^{1}$ \\ Kyoung Moo Choi, ${ }^{1}$ Andrew J. Haak, ${ }^{1}$ Aja Aravamudhan, ${ }^{1}$ Anja C. Roden, ${ }^{2}$ Y.S. Prakash, ${ }^{1,3}$ \\ Gwen Lomberk, ${ }^{4}$ Raul A. Urrutia, ${ }^{4}$ and Daniel J. Tschumperlin ${ }^{1}$ \\ 'Department of Physiology and Biomedical Engineering, ${ }^{2}$ Laboratory of Medicine and Pathology, and ${ }^{3}$ Department of \\ Anesthesiology, Mayo Clinic, Rochester, Minnesota, USA. ${ }^{4}$ Division of Research,Department of Surgery and Genomic \\ Sciences and Precision Medicine Center, Medical College of Wisconsin, Wauwatosa, Wisconsin, USA.
}

Pulmonary fibrosis is a devastating disease characterized by accumulation of activated fibroblasts and scarring in the lung. While fibroblast activation in physiological wound repair reverses spontaneously, fibroblast activation in fibrosis is aberrantly sustained. Here we identified histone 3 lysine 9 methylation (H3K9me) as a critical epigenetic modification that sustains fibroblast activation by repressing the transcription of genes essential to returning lung fibroblasts to an inactive state. We show that the histone methyltransferase G9a (EHMT2) and

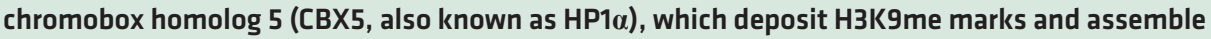
an associated repressor complex, respectively, are essential to initiation and maintenance of fibroblast activation specifically through epigenetic repression of peroxisome proliferatoractivated receptor $\gamma$ coactivator $1 \alpha$ gene (PPARGC1A, encoding PCC1 $\alpha$ ). Both TCF- $\beta$ and increased matrix stiffness potently inhibit PCC1 $\alpha$ expression in lung fibroblasts through engagement of the CBX5/C9a pathway. Inhibition of the CBX5/C9a pathway in fibroblasts elevates PCC1 $\alpha$, attenuates TCF- $\beta$ - and matrix stiffness-promoted H3K9 methylation, and reduces collagen accumulation in the lungs following bleomycin injury. Our results demonstrate that epigenetic silencing mediated by H3K9 methylation is essential for both biochemical and biomechanical fibroblast activation and that targeting this epigenetic pathway may provide therapeutic benefit by returning lung fibroblasts to quiescence.

Conflict of interest: The authors have declared that no conflict of interest exists.

Copyright: (c) 2019 American Society for Clinical Investigation

Submitted: January 2, 2019

Accepted: May 8, 2019

Published: June 20, 2019

Reference information: /CI Insight. 2019;4(12):e127111. https://doi. org/10.1172/jci.insight.127111.

\section{Introduction}

Pulmonary fibrosis is a devastating condition characterized by progressive accumulation of activated fibroblasts (sometimes referred to as myofibroblasts) and aberrant deposition of extracellular matrix (1-3). Activated fibroblasts arise in normal wound-healing responses to promote tissue repair; however, their sustained activation results in pathological scar formation $(4,5)$. While both biochemical and biomechanical stimuli have been found to drive fibroblast activation and aberrant matrix deposition (6-8), the mechanisms responsible for maintaining persistent and self-sustaining fibrogenesis remain incompletely characterized, posing significant barriers to the development of therapeutic interventions. A growing literature shows that epigenetic mechanisms may be responsible for perpetuating the activated state of fibroblasts by modulating the transcriptional activity of critical profibrotic genes involved in fibrosis progression (9-12). Epigenetic alterations, such as DNA methylation and histone modifications, play important roles in numerous physiological and pathological responses by regulating multiple biological processes, including cell differentiation, proliferation, and apoptosis (13-15). Altered epigenetic programs in diseased lung fibroblasts are known to contribute to idiopathic pulmonary fibrosis (IPF) (11, 16). While much attention has been given to the epigenetic activation of profibrotic gene expression during lung fibrosis development $(11,17,18)$, only recently epigenetic repression of antifibrotic genes has become appreciated as a potential mechanism that prevents the return of activated lung fibroblasts to a quiescent state (19-23).

Here, we identified the epigenetic repressor chromobox homolog 5 (CBX5) as a potentially novel regulator of fibroblast activation, acting via its transcriptional inhibitory functions to perpetuate the pathological activated state of diseased lung fibroblasts. CBX5 functions as a gene silencer that binds methylated 
lysine 9 residue on histone $3(\mathrm{H} 3 \mathrm{~K} 9 \mathrm{me})$, leading to the assembly of a transcriptional repressor complex (24-26). Our study reveals that CBX5 and the histone methyltransferase G9a (also known as EHMT2), responsible for methylating histone $3(\mathrm{H} 3)$ on lysine $9(27,28)$, are important mediators of lung fibroblast activation in response to both biochemical and mechanical stimuli in vitro, and in vivo we show that their functions within lung fibroblasts are essential to lung fibrogenesis.

Mechanistically, we found that CBX5 and G9a directly alter the chromatin landscape and repress transcription of the gene encoding PPAR $\gamma$ coactivator $1 \alpha(\mathrm{PGC} 1 \alpha)$, a master regulator of fatty acid oxidation and mitochondrial metabolism $(29,30)$. PGC1 $\alpha$ derepression is essential to the antifibrotic effects of CBX5/G9a inhibition, identifying PGC1 $\alpha$ as a potentially novel and potent regulator of fibroblast fate. Previous reports have shown that inhibition of PGC1 $\alpha$ in skeletal muscle leads to alterations in mitochondrial metabolism, including reduced oxidative phosphorylation (30). Interestingly, mitochondrial dysfunction has been shown to play a role in the development of IPF (31), suggesting that loss of mitochondrial gene expression due to epigenetic alterations may be important to the dysfunctional metabolism observed in this disease. Hence, our data shed light on G9a/CBX5/H3K9me as critical regulators of fibroblast activation and identify epigenetic repression of PGC1 $\alpha$ as a key mechanism promoting diseased lung fibroblast activation in support of lung fibrosis. Targeted inhibition of this epigenetic repression program may represent an important therapeutic strategy to reverse fibroblast activation and halt progressive lung fibrogenesis.

\section{Results}

Identification of $C B X 5$ as a potentially novel epigenetic regulator of fibroblast activation. To identify regulators of lung fibroblast activation during lung fibrosis progression, we recently completed a high-content siRNA screen of gene regulators of $\alpha \mathrm{SMA}^{+}$stress fibers in human lung fibroblasts (32). Through this screen we identified the epigenetic regulators BRD4, which has already been linked to fibroblast activation and lung fibrosis $(17,18,33)$, as well as CBX5, which has not previously been linked to fibroblast activation. Both of these epigenetic regulators are known for their interaction with $\mathrm{H} 3$ and for their role as global regulators of gene transcription $(34,35)$. BRD4 binds acetylated lysine 4 on $\mathrm{H} 3$, facilitating gene transcription, whereas CBX5 has high affinity for methylated lysine 9 (di-tri-methylated) on $\mathrm{H} 3$ and supports transcriptional repression. These findings suggest that contrasting positive and negative epigenetic control mechanisms may be critical for orchestrating fibroblast activation. While important roles for BRD4 and $\mathrm{H} 3 \mathrm{~K} 4$ acetylation-mediated gene activation are well established during fibrogenesis (36), the functions of the epigenetic repressor CBX5 and $\mathrm{H} 3 \mathrm{~K} 9$ methylation-mediated gene repression during lung fibrosis are not fully elucidated. To further investigate the role of CBX5 in fibroblast activation, we performed siRNA knockdown of CBX5 in IMR90 fibroblasts and evaluated the effect on TGF- $\beta$-induced profibrotic gene expression. CBX5 silencing significantly attenuated the expression of ACTA2, COL1A1, and FN1 (Figure 1A), hallmarks of fibroblast activation and ECM production. Similarly, Western blotting analysis demonstrated that CBX5 knockdown in TGF- $\beta$-treated lung fibroblasts blocked $\alpha$ SMA expression (Figure 1B). To relate profibrotic gene expression to de novo matrix synthesis and deposition, we adapted an antibody-based detection method to quantify fibroblast-deposited fibronectin and collagen I (37). We observed that CBX5 knockdown in lung fibroblasts strongly inhibited TGF- $\beta$-induced ECM protein deposition (Figure 1C). In order to evaluate whether CBX5 contributes to migratory responses, we performed a wound-healing assay and found that cell migration in the presence of TGF- $\beta$ was significantly impaired in CBX5-silenced fibroblasts compared with control cells (Figure 1D).

Prior work has demonstrated stable phenotypic alterations in fibroblasts isolated from patients with IPF, suggesting an epigenetic control of fibroblast activation (38-40). Therefore, we also performed siRNA-mediated knockdown of CBX5 in IPF-derived fibroblasts. Similar to our observation in TGF- $\beta$-stimulated normal fibroblasts, CBX5 knockdown significantly attenuated TGF- $\beta$ profibrotic functions in these diseased fibroblasts (Figure 1E). Interestingly, CBX5 knockdown significantly reduced profibrotic gene expression, even in absence of exogenous TGF- $\beta$ (Figure $1 \mathrm{~F}$ ), supporting a role for CBX5 in sustaining IPF-derived fibroblast activation during serial passage in vitro. Together, these findings indicate a broad relevance of CBX5 to ECM gene expression, matrix production, and cell migration in both TGF- $\beta$-stimulated and IPF-derived fibroblasts, consistent with an important role for this epigenetic repressor in initiating and sustaining fibroblast activation. Given that CBX5 behaves as transcriptional repressor and its inhibition blocks fibroblast activation, we hypothesized that CBX5 may directly contribute to the repression of genes whose function is critical to maintain or return fibroblasts to an inactive state (Figure $1 \mathrm{G}$ ). 
Inhibition of the histone methyltransferase G9a blocks biochemical and biomechanical fibroblast activation. CBX5 binds methylated lysine 9 on $\mathrm{H} 3$ (H3K9me), leading to the assembly of a transcriptional repressor complex that potently inhibits gene transcription (26). In addition to biochemical stimulation via TGF- $\beta$ leading to fibroblast activation, previous work has demonstrated that the pathological stiff environment present in fibrotic lungs also directly influences fibroblast activation $(6,7,41)$. Although the role of matrix stiffness in influencing the transcriptional machinery of lung fibroblasts to promote their activation is well established (42-44), the contribution of epigenetic remodeling and, more specifically, H3K9 methylation-mediated gene repression in regulating pathological mechanoresponses remain unexplored.

To evaluate whether matrix stiffness and TGF- $\beta$ directly influence global H3K9 methylation, we isolated primary mouse lung fibroblasts by FACS sorting and cultured them for 72 hours on soft or stiff substrates followed by immunofluorescence analyses using an antibody against $\mathrm{H} 3 \mathrm{~K} 9 \mathrm{me} 2 / 3$ marks as well as an antibody against $\alpha$ SMA to monitor cell activation. We found that lung fibroblasts cultured on stiff matrices displayed higher $\alpha$ SMA expression and elevated global H3K9 methylation compared with fibroblasts cultured on physiologically soft substrates (Figure 2A). To build on these observations, we compared the levels of global H3K9 methylation in normal human lung fibroblasts that have been cultured for 24 hours on soft $(0.2 \mathrm{kPa})$ or stiff (64 $\mathrm{kPa}$ and plastic) substrates in presence or absence of TGF- $\beta$. Western blotting analysis showed that the increased $\alpha \mathrm{SMA}$ expression in fibroblasts cultured on stiff substrates was accompanied by strong H3K9 methylation, and this effect was further amplified by the presence of TGF- $\beta$ in the culture media (Figure 2B).

$\mathrm{H} 3 \mathrm{~K} 9$ methylation (di- and trimethylation) is a mark of heterochromatin, a transcriptionally inactive state of chromatin $(25,27,45)$. Beside binding both di- and trimethylated lysine 9 on H3, CBX5 forms complexes with several others nonhistone proteins, including DNA methyltransferases $(46,47)$, histone methyltransferases (48), and histone deacetylases (HDACs) (49), which together with CBX5, participate in the repression of gene transcription. As we determined that both matrix stiffness and TGF- $\beta$ are important drivers of $\mathrm{H} 3 \mathrm{~K} 9$ methylation in lung fibroblasts, we reasoned that $\mathrm{H} 3 \mathrm{~K} 9$ me mark deposition by a histone methyltransferase may be critical to promote fibroblast activation in response to biomechanical or biochemical stimuli. We therefore cultured normal human lung fibroblasts on soft or stiff substrates for 24 hours in presence or absence of a small-molecule inhibitor (BIX01294) that specifically blocks the enzymatic activity the G9a histone methyltransferase (50), which is known to both bind CBX5 (48) and promote H3K9 methylation (28) and has recently been implicated in IPF fibroblast genetic repression (22, 51). BIX01294 treatment prevented matrix stiffness-induced $\alpha \mathrm{SMA}$ upregulation and reduced the levels of H3K9 methylation in lung fibroblasts (Figure 2C), suggesting that G9a-induced H3K9 methylation is required to promote fibroblast activation in response to biomechanical stimuli. While matrix stiffness stimulates both $\mathrm{H} 3 \mathrm{~K} 9 \mathrm{me} 2$ and $\mathrm{H} 3 \mathrm{~K} 9 \mathrm{me} 3$ mark deposition in lung fibroblasts, inhibition of G9a in these cells by BIX01294 reduced $\mathrm{H} 3 \mathrm{~K} 9 \mathrm{me} 2$ but had no effect on $\mathrm{H} 3 \mathrm{~K} 9 \mathrm{me} 3$ mark deposition, indicating that $\mathrm{H} 3 \mathrm{~K} 9$ dimethylation is directly implicated in mediating fibroblast mechanoresponses. Additionally, BIX01294 dose-dependently blocked $\alpha$ SMA expression following TGF- $\beta$ stimulation in normal lung fibroblasts plated on stiff plastic substrates (Figure 2D). Time-course experiments further confirmed the capacity of BIX01294 to block H3K9 dimethylation as early as 2 hours in lung fibroblasts cultured on stiff plastic substrates (Figure 2E).

Contractile force generation is an important function of activated fibroblasts $(52,53)$. We found that inhibition of G9a using siRNA significantly reduced cellular contractility, as demonstrated by traction force microscopy (Figure $2 \mathrm{~F}$ ) and by the collagen gel contraction assay (Figure $2 \mathrm{G}$ ), matching the effect of CBX5 siRNA. To extend our observations and evaluate the effect of inhibiting G9a in diseased fibroblasts, we treated IPF-derived fibroblasts with TGF- $\beta$ for 24 hours in presence or absence of BIX01294 or G9a siRNA. Both BIX01294 and G9a siRNA significantly reduced profibrotic gene expression, consistent with a critical role for G9a in maintaining the activated state of disease-derived cells (Figure 2, H and I). Taken together, these findings demonstrate that lung fibroblast $\mathrm{H} 3 \mathrm{~K} 9$ methylation is responsive to disease-relevant biochemical and biomechanical stimuli and that the histone methyltransferase G9a is essential to both responses. Moreover, inhibition of G9a in IPF-derived fibroblasts blunted their activation in the presence or absence of TGF- $\beta$, indicating that diseased fibroblasts remain responsive to pharmacologic targeting of $\mathrm{G} 9 \mathrm{a}$.

To investigate the direct disease relevance of these findings, we next probed G9a expression/localization in lung fibroblasts during experimental lung fibrogenesis in mice expressing Col1 1 1-GFP. Histological examination of the lungs from bleomycin-treated mice showed robust expansion of GFP-labeled cells compared with those from sham animals (Figure 3A). Additionally, a close examination of the lung tissue revealed increased expression of G9a in lung fibroblasts from bleomycin-treated lungs compared with lungs from sham 
A ACTA2

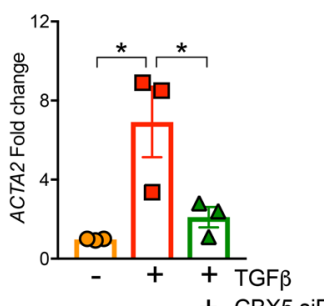

C
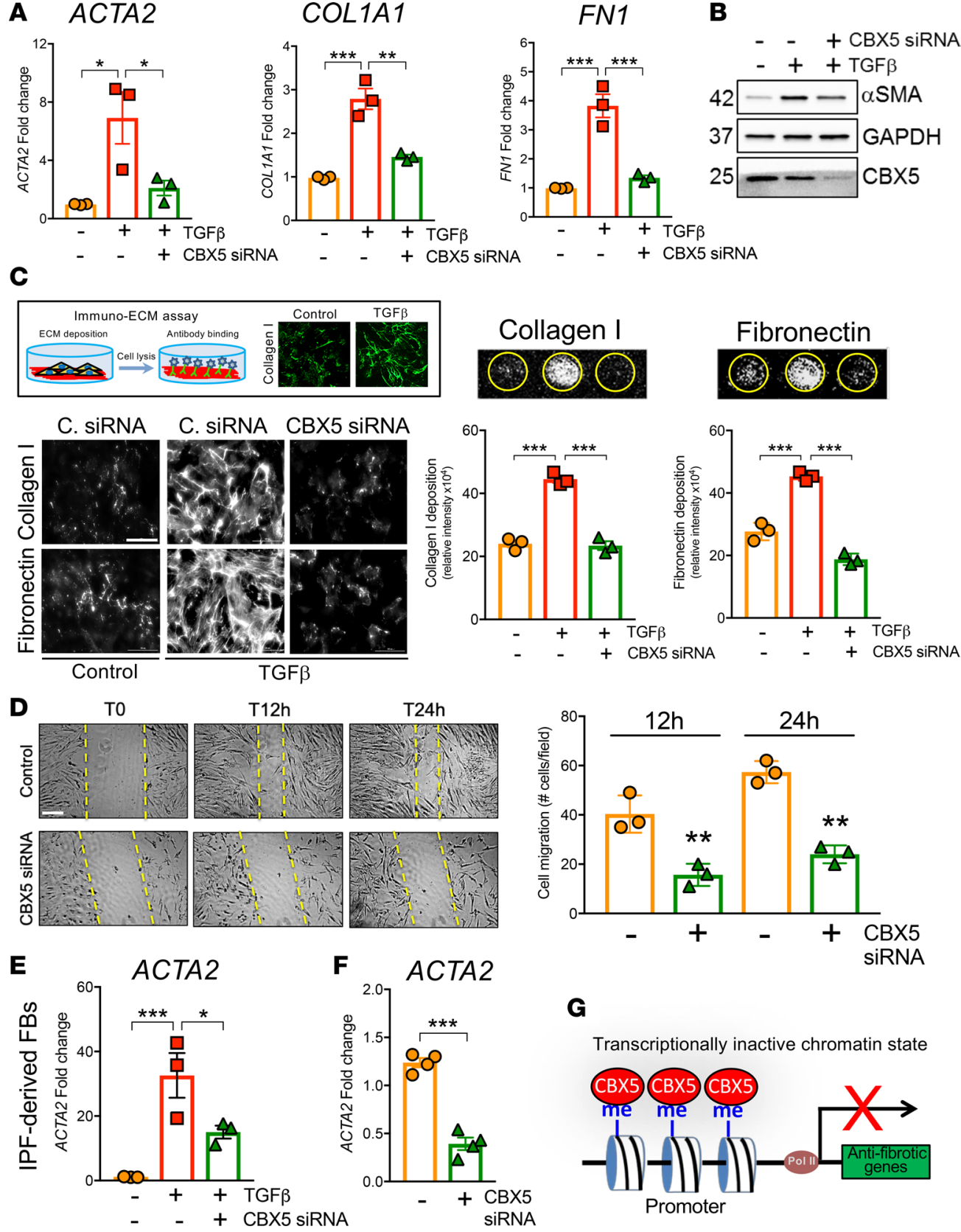

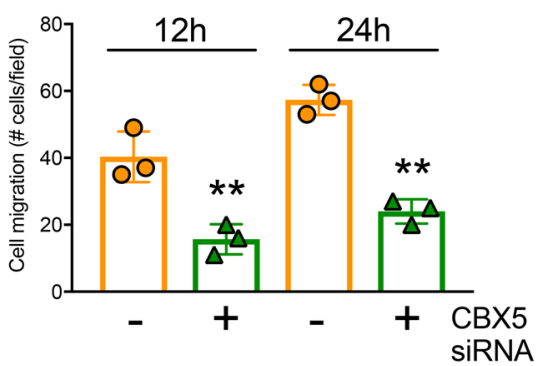

G

Transcriptionally inactive chromatin state

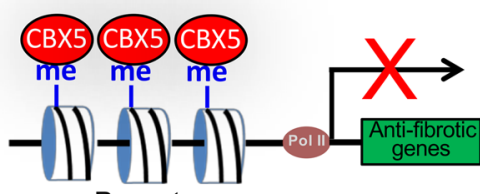

Promoter
Fibronectin

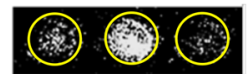

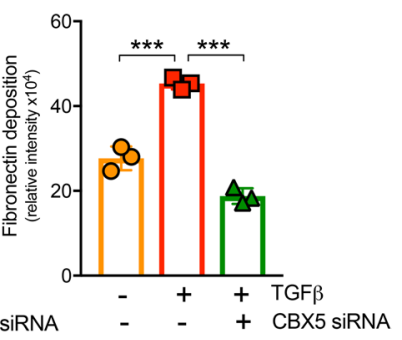

Figure 1. CBX5 silencing inhibits TGF- $\beta$-induced lung fibroblast activation. (A) IMR90 lung fibroblasts were transfected with CBX5 siRNA for 48 hours followed by TGF- $\beta$ stimulation for 24 hours. qPCR showed that TGF- $\beta$-stimulated profibrotic gene expression was significantly impaired in CBX5-silenced fibroblasts compared with those transfected with control siRNA $(n=3)$. Data are shown as mean \pm SEM of 3 independent experiments performed in duplicate. ( ${ }^{*} P<0.05$, ${ }^{* *} P<0.01$, ${ }^{* *} P<0.001$, by 1 -way ANOVA with Turkey's multiple comparisons test). (B) Western blotting showing that CBX5 knockdown in lung fibroblasts blocked TCF- $\beta$-stimulated $\alpha$ SMA expression (representative blot of $n=3$ ). (C) CBX5-silenced lung fibroblasts displayed deficient ECM protein deposition in response to TCF- $\beta$, as demonstrated by the immuno-ECM assay. Both fibronectin and collagen I were significantly reduced in CBX5-silenced fibroblasts compared with control cells. Data shown are representative of 3 independent experiments. Results are expressed as mean \pm SEM ( ${ }^{* * *} P<0.001$ by 2 -tailed, paired $t$ test). Scale bar: $10 \mu \mathrm{m}$. (D) Scratch assay shows reduced migratory capacity of CBX5-silenced lung fibroblasts in response to TGF- $\beta$ compared with control cells. Diminished cell migration was significant at 12 hours and remained impaired at 24 hours following TGF- $\beta$ exposure. Data represent the mean \pm SEM from 1 representative experiment performed in triplicate ${ }^{* *} P<0.01$ by 2 -tailed, paired $t$ test ). Scale bar: $10 \mu \mathrm{m}$. (E) qPCR analysis showing that siRNA knockdown of CBX5 in IPF-derived fibroblasts inhibits TCF- $\beta$-induced ACTA2 gene expression $(n=3)$. Data are shown as mean \pm SEM of 3 different IPF cell lines $\left({ }^{*} P<0.05,{ }^{* * *} P<0.001\right.$, by 1-way ANOVA with Turkey's multiple comparisons test). (F) ACTA2 gene expression is also significantly reduced in CBX5-silenced IPF fibroblasts in absence of TGF- $\beta(n=4)$. Data are shown as mean \pm SEM of 4 different IPF cell lines $\left({ }^{* *} P<0.001\right.$ by 2 -tailed, paired $t$ test). (G) Schematic representation showing interaction of CBX5 with methylated (me) histone 3 on proximal gene promoters. 
A
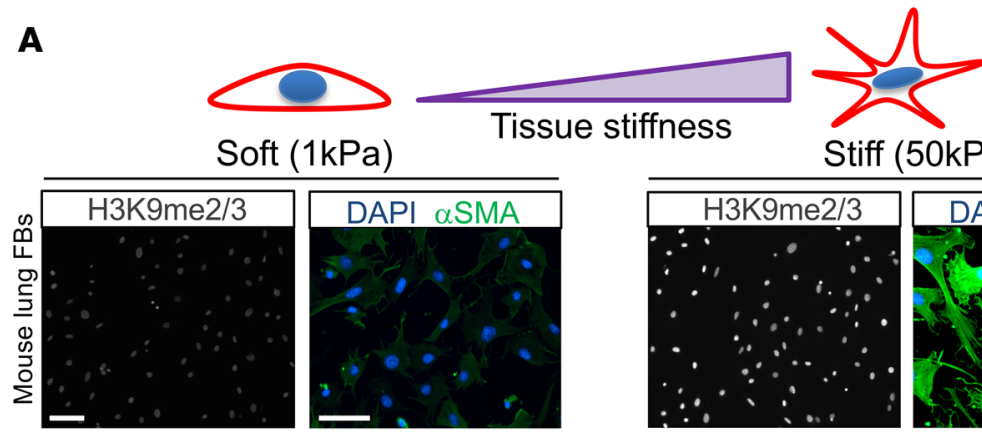

issue stiffness

Stiff (50kPa)

B

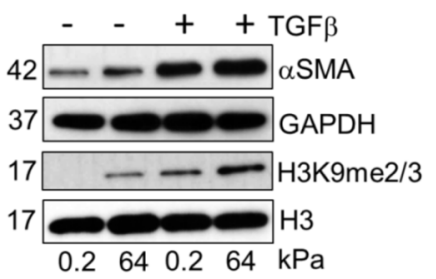

D
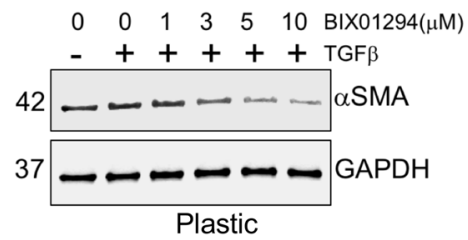

$\mathbf{F}$

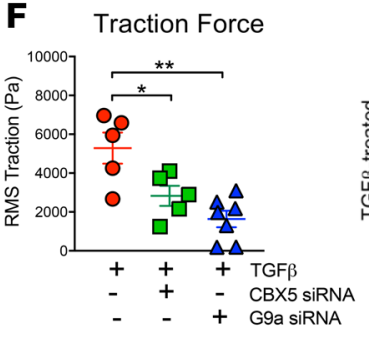

H

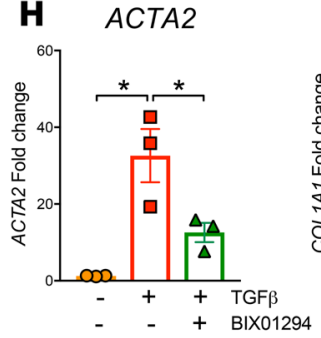

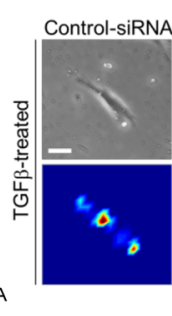

COL1A1

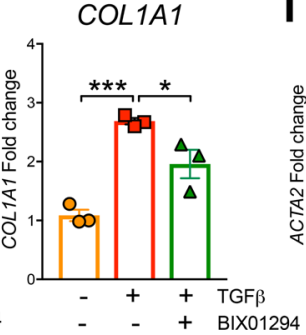

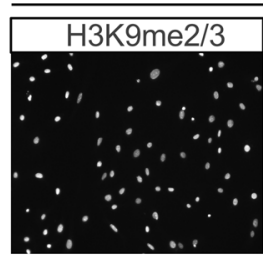

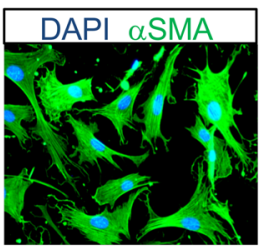

C

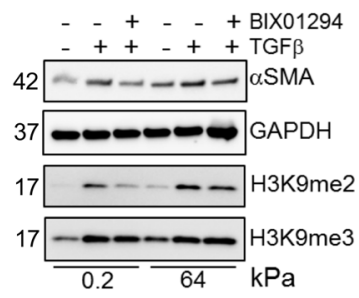

$\mathbf{E}$

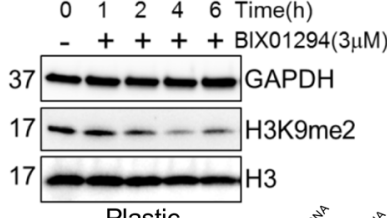

Plastic
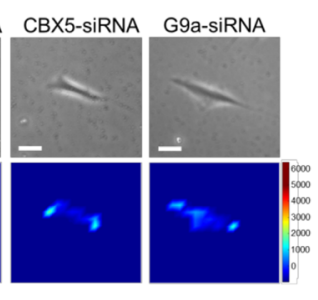

G
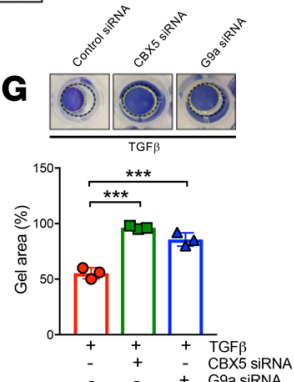

COL1A1
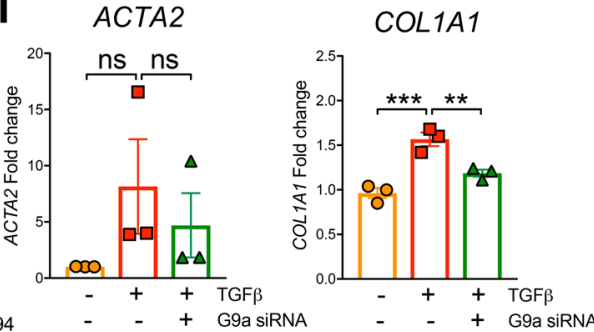

Figure 2. Matrix stiffness and TGF- $\beta$ stimulate global H3K9 methylation to promote lung fibroblast activation. (A) Representative IF images showing increased $\alpha \mathrm{SMA}$ expression and increased global H3K9me2/3 in freshly isolated mouse lung fibroblasts cultured on stiff hydrogels ( $50 \mathrm{kPa}$ ) compared with soft hydrogel (1 kPA) Scale bars: $20 \mu \mathrm{m}$. (B) Western blotting analysis showing increased H3K9me2/3 in fibroblasts seeded on stiff substrates (64 $\mathrm{kPa}$ ) compared with those seeded on soft substrates. H3K9 methylation was further increased by TCF- $\beta$ (representative blot of $n=3$ ). (C) Western blotting analysis shows that the G9a inhibitor BIX01294 inhibits matrix stiffness-promoted $\alpha$ SMA expression and H3K9me2 in human lung fibroblasts (representative blot of $n=2$ ). (D) BIX01294 dose-dependently blocks $\alpha$ SMA expression in human lung fibroblasts following TGF- $\beta$ stimulation (representative blot of $n$ = 3). (E) Western blotting analysis shows that inhibition of G9a time-dependently reduces global H3K9me2 in human lung fibroblasts (representative blot of $n=3)$. (F) CBX5 and C9a knockdown in human lung fibroblasts inhibits cell contractility, as measured by traction force microscopy $(n=5)$. $\left({ }^{*} P<0.05\right.$, ${ }^{* *} P<0.01$ by 2-tailed, paired $t$ test). Representative traction maps are shown. Scale bar: $10 \mu \mathrm{m}$. (C) Fibroblasts treated with CBX5 and G9a siRNA show reduced gel contraction compared with cells transfected with a control siRNA. Data shown are representative of 2 independent experiments performed in triplicate $(n=3)$. Results are expressed as mean \pm SEM ( ${ }^{* * *} P<0.001$ by 2 -tailed, paired $t$ test). (H) Gene expression analysis by qPCR shows that BIX01294 blocks ACTA2 and COL1A1 gene expression in IPF-derived fibroblasts in response to TCF- $\beta(n=3)$. Data are shown as mean \pm SEM of 3 different IPF cell lines ( ${ }^{*} P<0.05,{ }^{* *} P<0.001$ by 1 -way ANOVA with Turkey's multiple comparisons test). (I) Gene expression analysis by qPCR shows that knockdown of G9a in IPF-derived fibroblasts blocks TCF- $\beta$-stimulated COL1A1 gene expression and shows a trend toward reduced ACTA2 gene expression ( $n=3$ ). Data are shown as mean \pm SEM of 3 different IPF cell lines ( ${ }^{* *} P<0.01$, ${ }^{* *} P<0.001$ by 1-way ANOVA with Turkey's multiple comparisons test). 

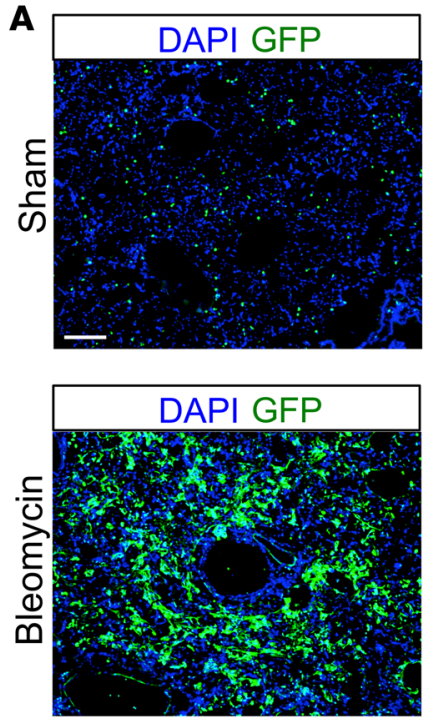

B

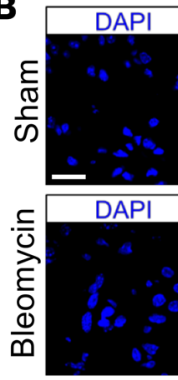

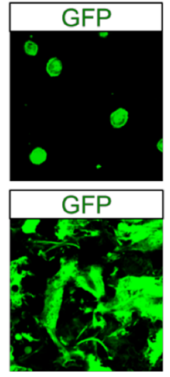
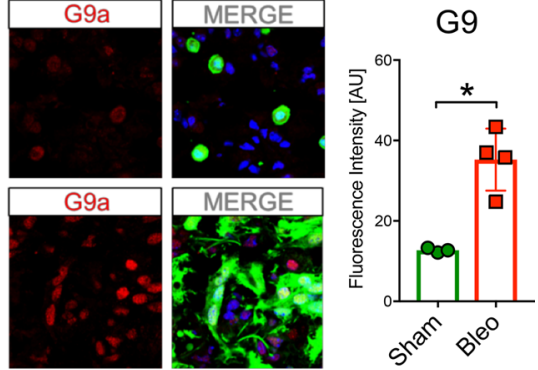

C

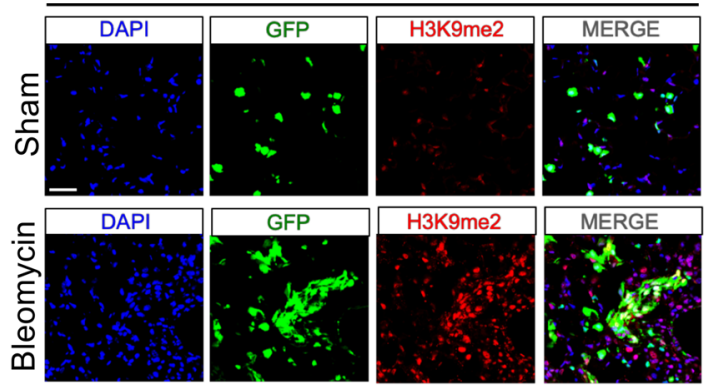

H3K9me2

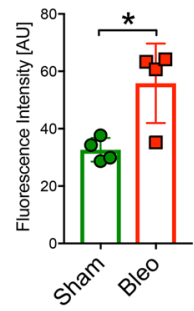

D

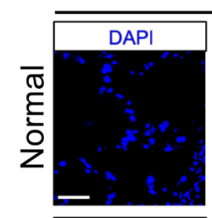

H3K9 methylation (Human tissue)

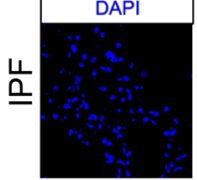

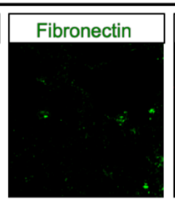

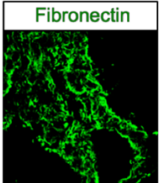

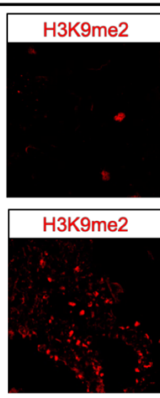
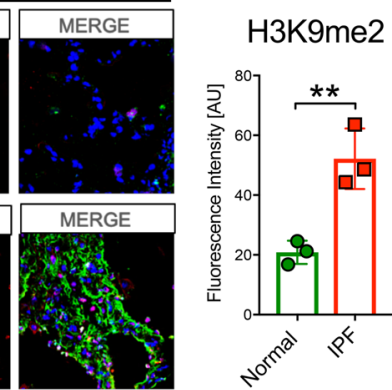

E

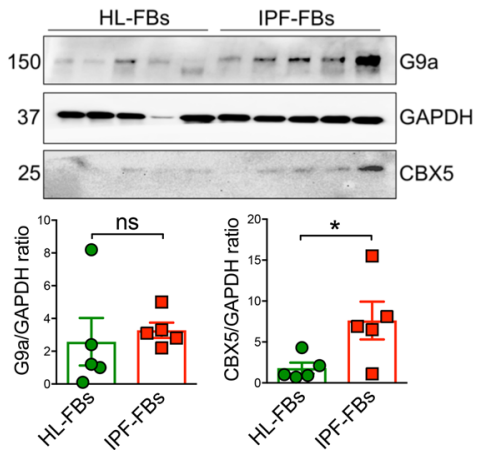

Figure 3. Nuclear G9a and H3K9 methylation are elevated in fibrotic lung tissues. (A) Lungs from Col1a1-CFP mice show GFP-labeled fibroblast expansion following bleomycin challenge (day 10) compared with control lungs. Scale bar: $100 \mu \mathrm{m}$. (B) Immunofluorescence images show increased G9a expression in $\mathrm{GFP}^{+}$fibroblasts from bleomycin-treated lungs compared with fibroblasts from healthy lungs. Fluorescence intensity was measured in GFP+ fibroblasts in normal and bleomycin-treated lung tissues using ImageJ software $(n=10)$. Data are expressed as mean \pm SEM of 3 normal and 4 bleomycin-treated lungs ( ${ }^{*} P<0.05$ by 2-tailed, paired $t$ test). Scale bar: $50 \mu \mathrm{m}$. (C) Immunofluorescence images showing elevated H3K9me2 in GFP+ fibroblasts from bleomycin-treated lungs compared with GFP+ fibroblasts from healthy lungs $(n=10)$. Data are expressed as mean \pm SEM of 4 normal and 4 bleomycin-treated lungs ( ${ }^{*} P<0.05$ by 2-tailed, paired $t$ test). Scale bar: $50 \mu \mathrm{m}$. (D) Immunofluorescence images showing increased H3K9me2 in lung tissues from IPF patients compared with healthy lungs $(n=10)$. Data are expressed as mean \pm SEM of 3 normal and 3 IPF lungs (** $P<0.01$ by 2 -tailed, paired $t$ test). Scale bar: $50 \mu \mathrm{M}$. (E) Western blotting showing CBX5 and G9a expression in IPF-derived and normal lung fibroblasts. Densitometry analysis demonstrated significant upregulation of CBX5 in IPF-derived fibroblasts compared with normal fibroblasts. No significant difference was observed in 69 a expression ( $n$ $=5$ ). Data are expressed as mean \pm SEM of 5 normal and 5 IPF-derived fibroblasts ( ${ }^{*} P<0.05$ by 2 -tailed, paired $t$ test).

animals (Figure 3B), suggesting that G9a may be involved in fibroblast activation during lung fibrogenesis. To extend these observations to H3K9 modifications, we stained both diseased mouse and human lung tissues with an antibody against H3K9 dimethylation and found strong immunostaining in GFP-labeled cells following bleomycin challenge compared with sham mice (Figure 3C). Similarly, we observed strong colocalization of fibronectin and H3K9 dimethylation in fibrotic regions of IPF lungs (Figure 3D), supporting potential relevance of G9a in the development and progression of IPF. Next, we evaluated G9a and CBX5 expression by Western blotting in IPF-derived fibroblasts relative to healthy fibroblasts and found that while there was no statistical difference in G9a, CBX5 expression was significantly elevated in IPF fibroblasts compared with normal ones, suggesting a role for CBX5 in sustaining fibroblasts activation ex vivo (Figure 3E).

In vivo-activated lung fibroblasts are characterized by enhanced ECM deposition and stable repression of peroxisomal genes. Given that the CBX5/G9a pathway plays a role in epigenetically repressing gene transcription (48), we hypothesized that this epigenetic complex must repress expression of genes that 
actively maintain or restore the quiescent state of lung fibroblasts. Rather than relying solely on in vitro activation studies to identify disease-relevant fibrosis suppressor genes, we employed the bleomycin model to generate fibrosis in mice to study the activated state of freshly isolated lung fibroblasts. To identify the fibroblast population of the mouse lungs, we again used the Col1 1-GFP-transgenic mouse, in which cells with a transcriptionally active Col1 $\alpha 1$ promoter are GFP labeled. In addition, antibodies against known markers for leukocytes (CD45), endothelial cells (CD31), and epithelial cells (CD326) were also included in the FACS sorting to further ensure an optimal separation of the fibroblast from other cell populations of the lung (Figure 4A). Two weeks following bleomycin administration, diseased and normal lungs were harvested, minced to generate a single-cell suspension, and subjected to FACS. To identify genes whose expression is reduced during fibroblast activation, we evaluated the expression of genes in FACS-isolated lung fibroblasts from sham- and bleomycin-treated animals. Based on an extensive literature linking peroxisomal genes to inhibition of fibroblast activation (54-57), we focused on family members PPAR $\alpha, \operatorname{PPAR} \gamma$, and their coregulator PGC1 $\alpha$. In freshly isolated lung fibroblasts from bleomycin-treated mice we observed significant upregulation of $F n 1$ gene expression and strong repression of Ppara and Ppargcla genes compared with lung fibroblasts from sham animals. Surprisingly, despite the established role of PPAR $\gamma$ in fibrosis (57), we did not observe a significant difference in the expression of this gene between bleomycin-treated and normal lung fibroblasts (Figure 4B). To assess whether the disease-activated state is maintained in vitro, freshly isolated $\mathrm{GFP}^{+}$lung fibroblasts from sham- and bleomycin-treated mice were cultured for 1 week followed by Western blotting analysis to evaluate pro-and antifibrotic gene expression. As shown in Figure 4C, lung fibroblasts isolated from bleomycin-treated mice displayed strong $\alpha$ SMA expression and sustained differences in PPAR $\alpha$ and PGC1 $\alpha$ protein levels relative to fibroblasts from sham-treated mice. In addition, in situ ECM deposition measurements revealed that in vivo-activated fibroblasts generate significantly more collagen than those isolated from healthy lungs after 1 week in culture. Inhibition of G9a by BIX01294 reduced collagen deposition in these cells to a level that was comparable to control healthy fibroblasts (Figure 4D), demonstrating their responsiveness to G9a inhibition. To assess whether inhibition of G9a in vivo using BIX01294 restored/elevated peroxisomal gene expression in lung fibroblasts following lung injury, we treated mice with bleomycin and 10 days following instillation we i.p. injected them with a single dose of BIX01294 ( $5 \mathrm{mg} / \mathrm{kg}$ ) followed by lung fibroblast isolation by FACS sorting (Figure 4E). In order to evaluate the efficacy of BIX01294 in blocking H3K9 methylation in vivo we set up a parallel experiment in which global H3K9 methylation was evaluated in whole lung by Western blotting at 6, 24, and 48 hours following the delivery of a single dose of BIX01294. As shown in Figure 4F, BIX01294 treatment reduced global H3K9 methylation in whole lung compared with vehicle, and this effect was detectable at 24 hours and 48 hours following BIX01294 injection. Additionally, qPCR analysis showed elevated Ppargcla and Ppara gene expression in fibroblasts isolated from the lungs of BIX01294-treated animals compared with DMSO-treated control lungs (Figure 4G), demonstrating that pharmacological manipulation of epigenetic mechanisms restored peroxisomal gene expression in diseased lung fibroblasts, potentially altering their fibrogenic behavior.

Inhibition of CBX5 in activated lung fibroblasts restores peroxisomal gene expression. To test whether peroxisomal proteins are acutely regulated by TGF- $\beta$, we measured each by Western blotting and confirmed TGF- $\beta$-mediated reductions in expression in both normal and IPF-derived fibroblasts (Figure 5, A and C). To test the role for CBX5 in TGF- $\beta$-mediated peroxisomal gene repression, we compared cells treated with CBX5 siRNA (48 hours) followed by TGF- $\beta$ treatment for 24 hours. Transcripts for all 3 peroxisomal genes were potently repressed by TGF- $\beta$, and this repression was prevented by CBX 5 siRNA (Figure 5, B and D). To extend these observations to matrix stiffness-mediated fibroblast activation, we cultured normal lung fibroblasts on soft/ stiff substrates for 24 hours and evaluated peroxisomal gene expression by qPCR. Interestingly, fibroblasts cultured on pathologically stiff substrates showed strong inhibition of both PPARGC1A and PPARA gene expression but not $P P A R G$ gene expression compared with those cultured on substrates of normal compliance (Figure 5E). Inhibition of CBX5 by siRNA in fibroblasts cultured on stiff substrates fully restored PGC1 $\alpha$ and PPAR $\alpha$ expression (Figure 5F), demonstrating that this epigenetic reader is essential to matrix stiffness-mediated peroxisomal gene repression. Together, our results demonstrate that CBX5 mediates repression of putative fibrosis-suppressor peroxisomal genes in response to TGF- $\beta$ and matrix stiffness and the same genes are repressed in vivo in fibroblasts during experimental lung fibrosis (Figure 4B). Interestingly, inhibition of the other epigenetic regulator identified in our prior screen, BRD4, was similarly effective in reducing profibrotic 

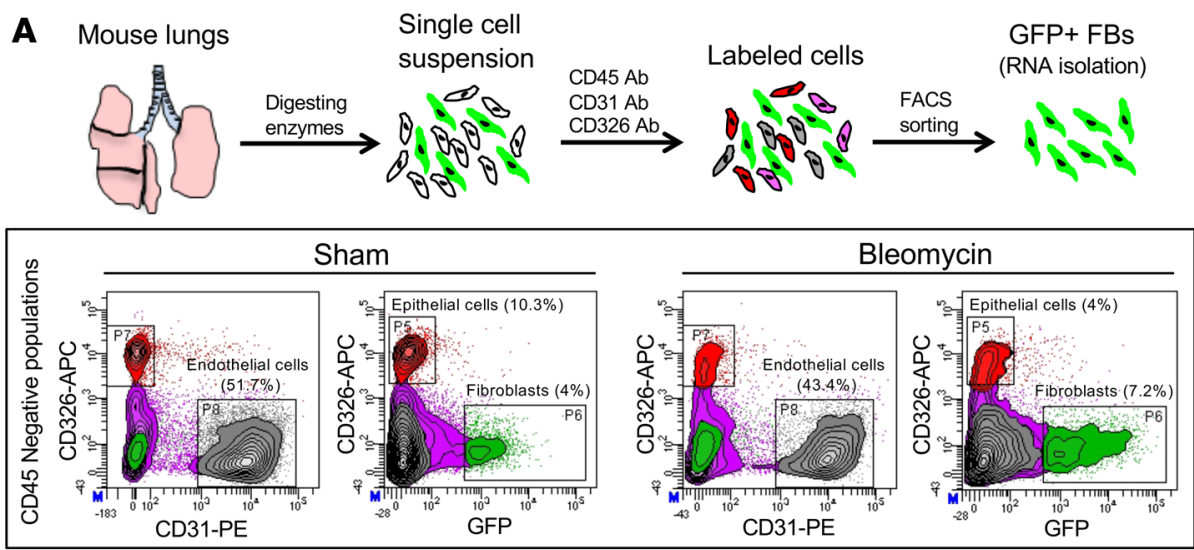

B Non-cultured GFP+ FBs

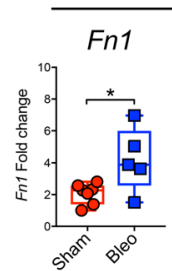

Ppargc1a

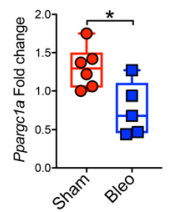

Ppara
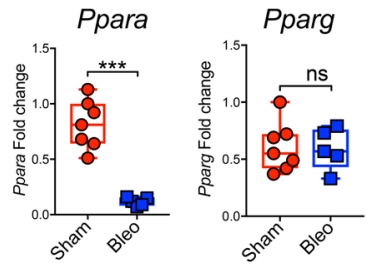

C

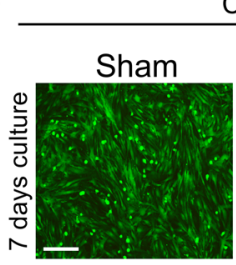

Cultured GFP+ FBs

D

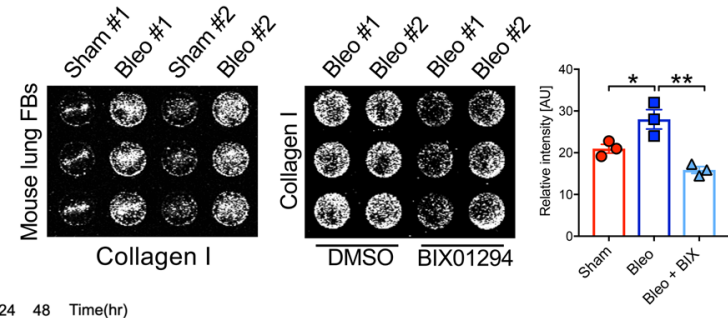

$\mathbf{E}$
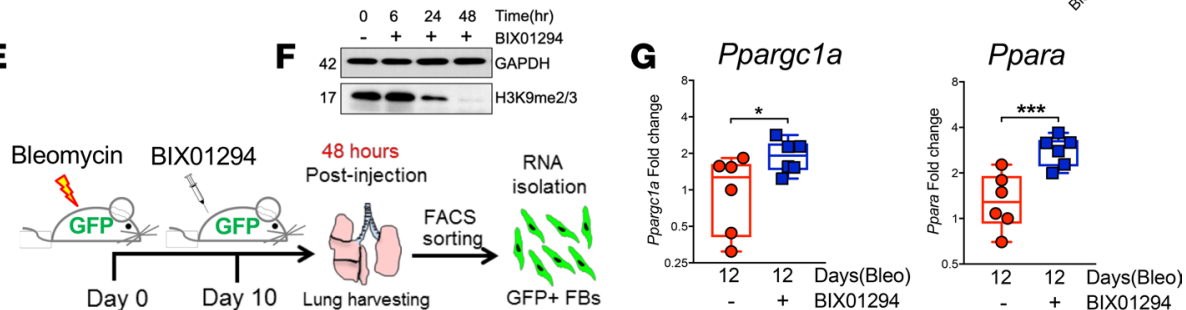

Figure 4. Fibroblasts isolated from fibrotic lungs display sustained peroxisomal gene repression in vitro. (A) Lungs from bleomycin- or vehicle-treated Col1a1-GFP mice were harvested 2 weeks following bleomycin administration. FACS sorting was employed to isolate GFP+ fibroblasts from diseased and healthy lungs. Antibodies against markers for epithelial (CD326), endothelial cells (CD31), and leukocytes (CD45) were included in the analysis. Gating strategy includes exclusion of CD45+ cells, doublets and dead cells (data not shown). FACS analysis reveals an increased number of GFP+ fibroblasts and higher GFP intensity in fibroblasts from diseased lungs compared with healthy lungs. (B) qPCR on FACS-sorted lung fibroblasts shows elevation of Fn1 gene expression and repression of peroxisomal genes, including Ppara and Ppargc1a, but not Pparg, in diseased compared with normal fibroblasts $(n=5)$. Results are expressed as mean \pm SEM $\left({ }^{*} P<0.05\right.$, ${ }^{* * *} P<0.001$ by 2 -tailed, paired $t$ test). (C) Representative images showing cultured GFP+ fibroblasts from bleomycin-treated or healthy lungs. Scale bar: $10 \mu \mathrm{m}$. Wester blotting showing elevated $\alpha$ SMA expression and sustained PGC1 $\alpha$ and PPAR $\alpha$ repression in cultured GFP+ lung fibroblasts from diseased lungs compared with healthy GFP+ fibroblasts (Sham, $n=2$; Bleo, $n=2$ ). (D) Immuno-ECM assay analysis shows that diseased mouse lung fibroblasts display increased collagen I deposition compared with normal ones. Inhibition of G9a by BIX01294 in diseased fibroblasts significantly reduces collagen I deposition to a level comparable to normal fibroblasts. Results are expressed as mean \pm SEM $\left({ }^{*} P<0.05,{ }^{* *} P<0.01\right.$ by 1 -way ANOVA with Turkey's multiple comparisons test). (E) Experimental strategy to evaluate peroxisomal gene expression in diseased lung fibroblasts in vivo following G9a inhibition by BIX01294. (F) Western blotting image showing reduced H3K92/3me in whole lung at 24 and 48 hours following BIX01294 administration (representative blot of $n=2$ ). (C) qPCR shows Ppargc1a and Ppara elevation in fibroblasts from BIX01294-treated mice compared with control mice ( $n$ $=6)$. Results are expressed as mean $\pm \operatorname{SEM}\left({ }^{*} P<0.05,{ }^{* * *} P<0.001\right.$ by 2 -tailed, paired $t$ test $)$. 
gene expression, but had the opposite effect on PPARGC1A relative to G9a inhibition (Supplemental Figure 1; supplemental material available online with this article; https://doi.org/10.1172/JCI12345DS). This result confirms the overall important roles of epigenetic regulators in fibroblast activation but also emphasizes the likelihood that their specific effects on fibroblast fate beyond reducing canonical activation genes may be distinct and important.

PGC1 $\alpha$ plays a leading role as a fibrosis-suppressor gene in lung fibroblasts. To determine whether inhibition of G9a-induced H3K9 methylation by BIX01294 replicated the results obtained with CBX5 silencing in lung fibroblasts, we first cultured IPF-derived fibroblasts in presence or absence of BIX01294 for 2, 4, and 6 hours followed by Western blotting analysis to evaluate global H3K9 methylation. As shown in Figure 6A, BIX01294 reduced H3K9 methylation starting at 2-4 hours after exposure. This rapid kinetic profile prompted us to evaluate the effects of BIX01294 on TGF- $\beta$-stimulated profibrotic gene expression during the first 12 hours following TGF- $\beta$ treatment. BIX01294 inhibited TGF- $\beta$-induced ACTA2 and COL1A1 gene expression at 12 hours (Figure 6B) and blocked TGF- $\beta$-mediated PPARGC1A gene repression starting at 4 hours (Figure 6C), demonstrating that BIX01294-mediated PPARGC1A elevation preceded the reduction of profibrotic genes. Intriguingly, BIX01294 failed to restore PPARA and PPARG gene expression at these time points, suggesting that these peroxisomal genes might not directly contribute to the antifibrotic effects of BIX01294 (Figure 6C). Furthermore, BIX01294 significantly elevated PPARGC1A gene expression at 8 hours and blocked $A C T A 2$ gene expression at 12 hours in IPF fibroblasts that have not been treated with TGF- $\beta$ (Figure 6D), confirming similar kinetics and therapeutic sensitivity in disease-derived fibroblasts. Taken together, these observations suggest a potential leading role for PPARGC1A gene activation in attenuating fibroblast activation downstream of G9a inhibition.

To rule out the involvement of PPAR $\gamma$ and PPAR $\alpha$ in mediating the antifibrotic effects of inhibiting G9a or CBX5 in lung fibroblasts, we individually blocked their activity using either small-molecule antagonists or siRNAs in presence or absence of BIX01294 or CBX5 siRNA. Neither an antagonist of PPAR $\gamma$ nor PPAR $\gamma$ siRNA prevented the BIX01294- or CBX5 siRNA-mediated inhibition of ACTA2 gene expression in these cells, demonstrating that the beneficial effects of inhibiting CBX5/G9a are PPAR $\gamma$ independent (Figure 6E). Moreover, while PPAR $\alpha$ siRNA was partially effective (Figure 6F), PGC1 $\alpha$ siRNA fully reversed the CBX5 siRNA- and BIX01294-mediated inhibition of ACTA2 gene expression (Figure 6G) and reversed the CBX5 siRNA-mediated inhibition of $C O L 1 A 1$ gene expression in responses to TGF- $\beta$ (Figure $6 \mathrm{H})$. These results confirm that repression of PGC1 $\alpha$, in a CBX5/G9a-dependent fashion, is an early and essential event in TGF- $\beta$-induced lung fibroblast activation.

CBX5 directly interacts with the PPARGC1A gene promoter. Having demonstrated the key role of CBX5 and H3K9 methylation in promoting PPARGC1A gene repression, we sought to establish their direct interactions with the PPARGC1A gene promoter. To first evaluate whether CBX5 binds PPARGC1A gene proximal promoter we carried out a ChIP analysis and assayed CBX5 binding at 3 different regions of the PPARG$C 1 A$ gene promoter that were previously shown to contain consensus sequences for numerous transcription factors (29). ChIP analysis demonstrated binding of CBX5 to all 3 regions and also revealed that this interaction was further increased by TGF- $\beta$ (Figure 7A) and reduced by CBX5 siRNA (Supplemental Figure 2). Similarly, $\mathrm{H} 3 \mathrm{~K} 9 \mathrm{me} 2$ mark enrichment was also increased at the same genomic locations as a result of TGF- $\beta$ stimulation, and this enrichment was attenuated in all 3 locations by BIX01294, thereby confirming that TGF- $\beta$-mediated H3K9 methylation at PPARGC1A gene promoter is dependent on G9a activity (Figure 7B). These findings delineate a potentially novel mechanism by which TGF- $\beta$ induces epigenetic remodeling of $P P A R G C 1 A$ gene promoter to facilitate its repression and promote fibroblast activation.

Two of the CBX5-binding sites $(-159+85$ and $-377-282)$ on the PPARGC1A gene promoter are positioned in close proximity to the transcription starting site and contain known consensus binding sites for the cAMP response element-binding transcription factor CREB (29). CREB is well known to be activated by the cAMP/PKA signaling pathway to promote the transcription of numerous genes (58). Interestingly, previous publications reported deficient activation of the CAMP/PKA/CREB signaling pathway in fibroblasts isolated from fibrotic lungs (59). To explore the relevance of CREB in the repression of PGC1 $\alpha$ by TGF- $\beta$ and link H3K9 methylation to the cAMP/PKA signaling pathway, we inhibited both CBX5 and CREB in TGF- $\beta$-stimulated lung fibroblasts. Inhibition of CBX 5 alone promoted PPARGC1A gene expression in TGF- $\beta$-treated fibroblasts, while knockdown of CREB in these cells blocked this transcriptional elevation (Figure 7C). In addition, similarly to what we observed in PGC1 $\alpha$-silenced lung fibroblasts, CREB knockdown elevated ACTA2 gene expression and partially blocked the antifibrotic effects of inhibiting 
A

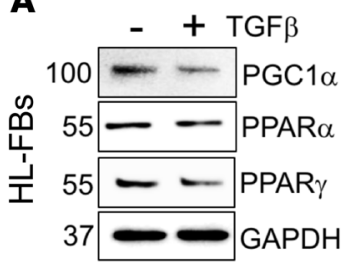

C

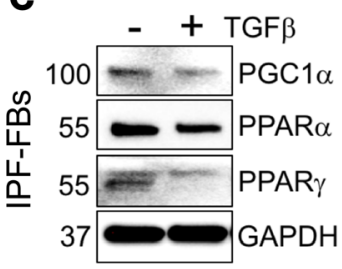

B PPARGC1A

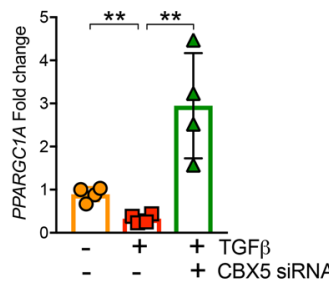

D PPARGC1A

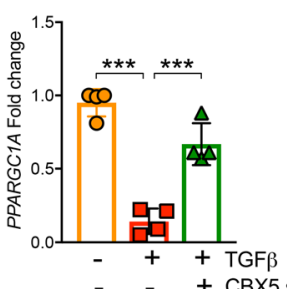

PPARG

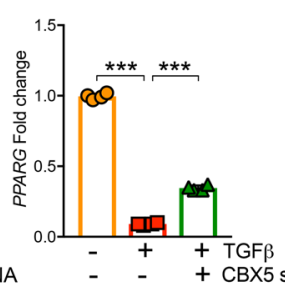

PPARG

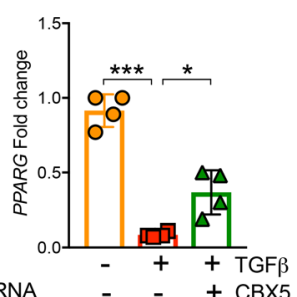

PPARA

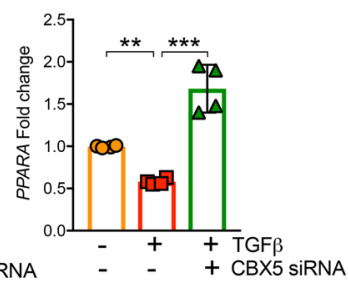

PPARA

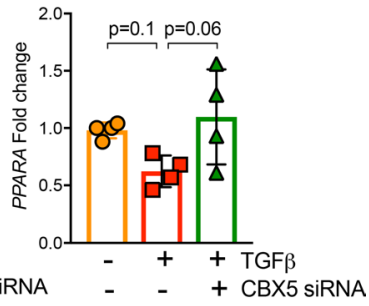

E Soft Pro-fibrotic genes Stiff

PPARGC1A

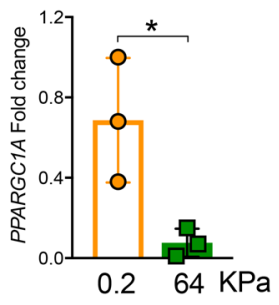

PPARA

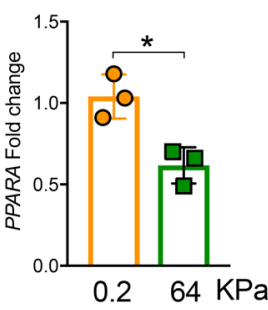

PPARG

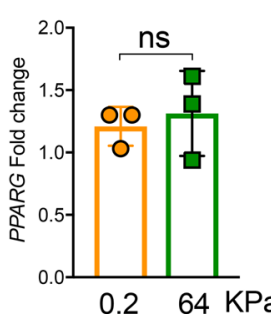

F - - + CBX5 SiRNA

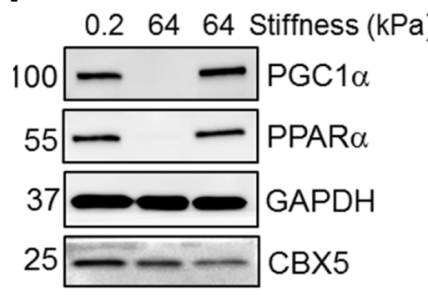

Figure 5. Inhibition of CBX5 in activated lung fibroblasts promotes upregulation of peroxisomal genes in vitro. (A) Western blotting analysis shows that TGF- $\beta$ potently inhibits peroxisomal protein expression in normal human lung fibroblasts (representative blot of $n=3$ ). Cells were incubated with TCF- $\beta$ for 24 hours. (B) qPCR analysis reveals that inhibition of CBX5 in normal human lung fibroblasts prevents TCF- $\beta$-inhibited peroxisomal gene expression $(n=3)$. Data are shown as mean \pm SEM of 3 independent experiments performed in duplicate. $\left({ }^{*} P<\right.$ $0.05,{ }^{* *} P<0.01,{ }^{* *} P<0.001$ by 1 -way ANOVA with Turkey's multiple comparisons test). (C) Wester blotting analysis shows strong repression of peroxisomal proteins by TCF- $\beta$ in IPF-derived fibroblasts (representative blot of $n$ = 3). (D) Similarly to normal lung fibroblasts, inhibition of CBX5 in IPF-derived fibroblasts blocks TGF- $\beta$-inhibited peroxisomal gene expression $(n=3)$. Data are shown as mean \pm SEM of 3 independent experiments performed in duplicate $\left({ }^{*} P<0.05,{ }^{* *} P<0.001\right.$ by 1 -way ANOVA with Turkey's multiple comparisons test). (E) Gene expression analysis shows PPARA and PPARGC1A gene repression in normal lung fibroblasts seeded on stiff substrates (64 $\mathrm{kPa}$ ) compared with soft $(0.2 \mathrm{kPa})(n=3)$. Data are shown as mean $\pm \mathrm{SEM}$ of 3 independent experiments performed in duplicate ( ${ }^{*} P<0.05$ by 2-tailed, paired $t$ test). (F) Western blotting analysis shows downregulation of PPAR $\alpha$ and PGC1 $\alpha$ in lung fibroblasts cultured on stiff compared with soft substrates. CBX5 silencing blocks matrix stiffness-mediated PPAR $\alpha$ and PGC1 $\alpha$ downregulation in lung fibroblasts representative blot of $(n=2)$.

CBX5 in these activated cells (Figure 7D). These findings identify CREB as an important transcription factor upstream of PGC1 $\alpha$ and reveal that inhibition of the CBX5/G9a pathway in activated lung fibroblasts facilitates CREB-mediated PPARGC1A transcription to attenuate fibrogenic cell activation. Based on the altered $\mathrm{H} 3 \mathrm{~K} 9$ methylation observed in fibrotic lung tissues, we expect that $\mathrm{CBX} 5$ genomic residence during lung fibrosis development may inhibit the interaction of CREB with the PPARGC1A gene promoter, resulting in reduced gene transcription, thereby supporting ongoing fibroblast activation in vivo (Figure 7E).

Inhibition of G9a restores Ppargcla gene expression in vivo and attenuates fibrosis progression. We previously showed that G9a inhibitor BIX01294 acutely elevates fibroblast Ppargc1a expression in vivo (Figure 4G). To test whether inhibition of G9a by BIX01294 is capable of stably elevating Ppargcla gene expression in the lung and altering the trajectory of experimental fibrosis, we treated mice with bleomycin followed by intraperitoneal administration of a single dose of BIX01294 $(5 \mathrm{mg} / \mathrm{kg}$ ) or vehicle, delivered at day 10 (Figure 8A). Ten days following BIX01294 treatment lungs were harvested, and $\mathrm{qPCR}$ was performed to assess 
A

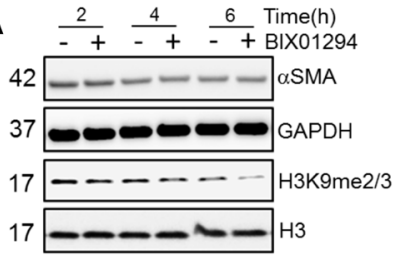

C

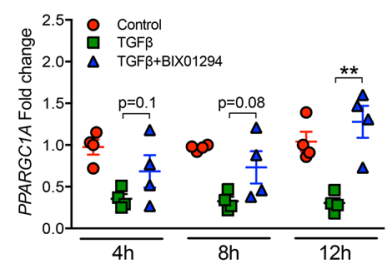

D

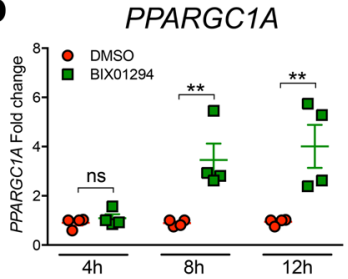

E

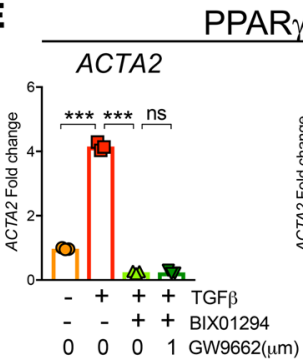

G
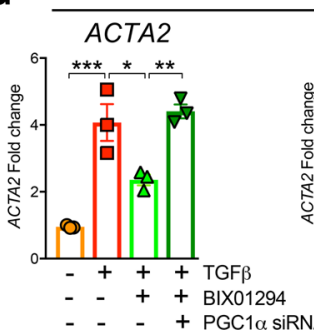

B

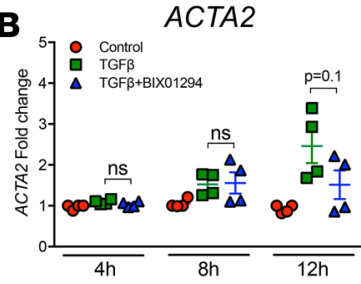

PPARA

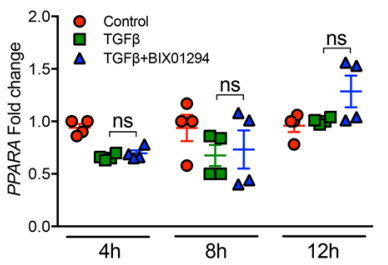

ACTA2

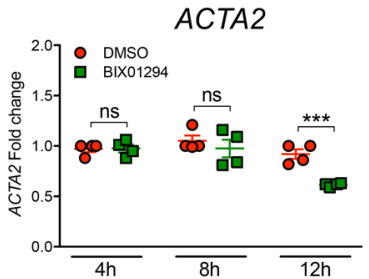

COL1A1

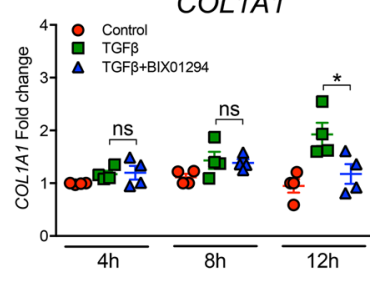

PPARG

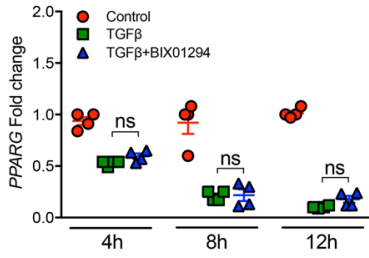

COL1A1

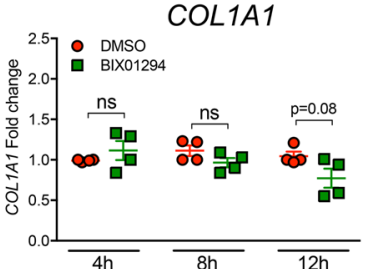

$\mathbf{F}$

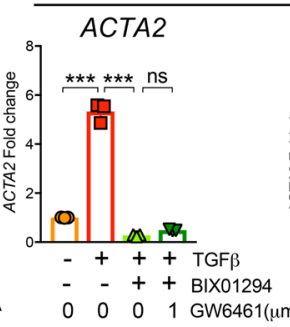

H

PGC10

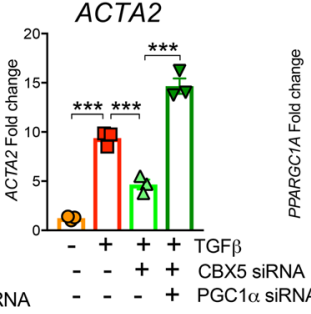

PPARGC1A

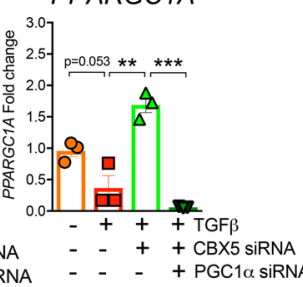

Figure 6. PGC1 $\alpha$ inhibition by the G9a/CBX5 pathway is an early and necessary step in TGF- $\beta$-mediated fibroblast activation. (A) Western blotting showing reduction of $\mathrm{H} 3 \mathrm{~K} 9 \mathrm{me} 2 / 3$ in IPF-derived fibroblasts exposed to BIX01294 for 2, 4, and 6 hours (representative blot of $n=2$ ). (B) qPCR of IPF-derived fibroblasts treated with TGF- $\beta$ shows an increase of ACTA2 and COL1A1 gene expression at 12 hours following TCF- $\beta$ exposure. BIX01294 treatment reduces ACTA2 and COL1A1 elevation by TGF- $\beta$ at 12 hours but has no effect at 4 or 8 hours. Data are shown as mean \pm SEM of 4 different IPF cell lines ( ${ }^{*} P<0.05$ by 2 -tailed, paired $t$ test). (C) qPCR analysis showing that TGF- $\beta$ inhibits PPARA, PPARG, and PPARGC1A gene expression starting at 4 hours following treatment. BIX01294 treatment prevents PPARGC1A gene repression by TGF- $\beta$, without significant effect on PPARA and PPARG. Data are shown as mean \pm SEM of 4 different IPF cell lines (**P 0.01 by 2 -tailed, paired $t$ test). (D) BIX01294 significantly reduces ACTA2 gene expression and elevated PPARGC1A gene transcripts. Data are shown as mean \pm SEM of 4 different IPF cell lines $\left({ }^{* *} P<0.01,{ }^{* *} P<0.001\right.$ by 2 -tailed, paired $t$ test). (E and F) PPAR $\gamma$ or PPAR $\alpha$ inhibition by siRNA or antagonists in BIX01294-treated of CBX5-silenced fibroblasts fails to block the beneficial effects of inhibiting G9a or CBX5 in TGF- $\beta$-treated fibroblasts. (G) Inhibition of PGC1 $\alpha$ by siRNA in BIX01294-treated or CBX5-silenced fibroblasts restores the elevation of ACTA2 by TCF- $\beta$. Data are shown as mean \pm SEM of 3 independent experiments performed in duplicate $\left({ }^{*} P<0.05,{ }^{* *} P<0.01,{ }^{* *} P<0.001\right.$ by 1 -way ANOVA with Turkey's multiple comparisons test). (H) qPCR analysis showing that inhibition of PGC1 $\alpha$ in CBX5-silenced fibroblasts promotes COL1A1 gene elevation in response to TGF- $\beta$. Data are shown as mean \pm SEM of 4 independent experiments performed in duplicate ( ${ }^{*} P<0.05$ by 1 -way ANOVA with Turkey's multiple comparisons test). 
A
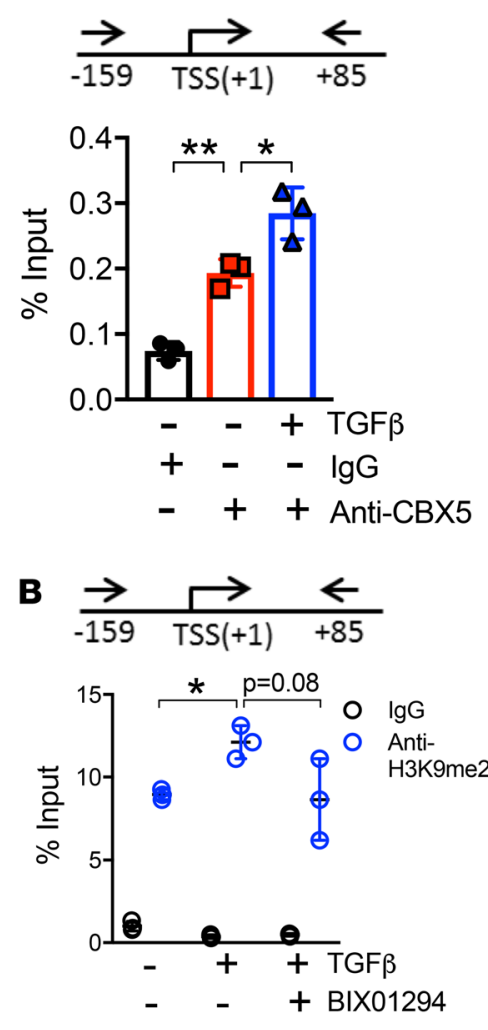

C

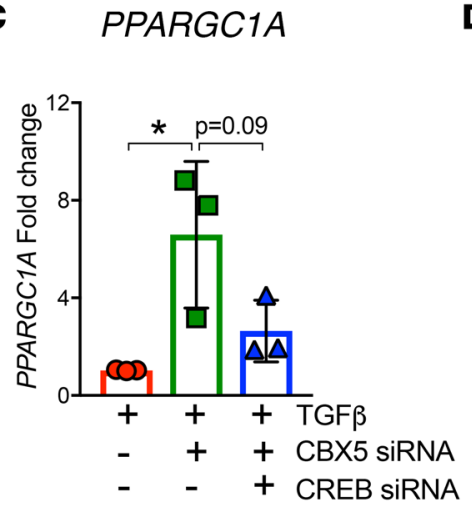

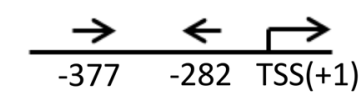
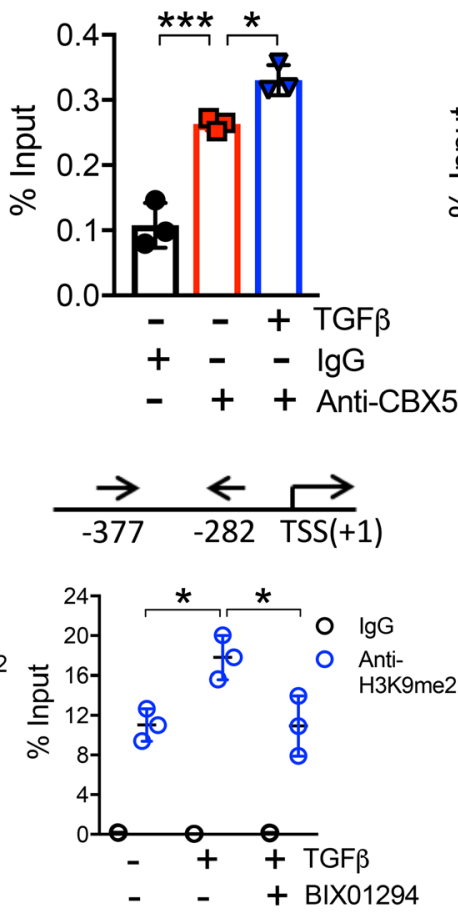

D

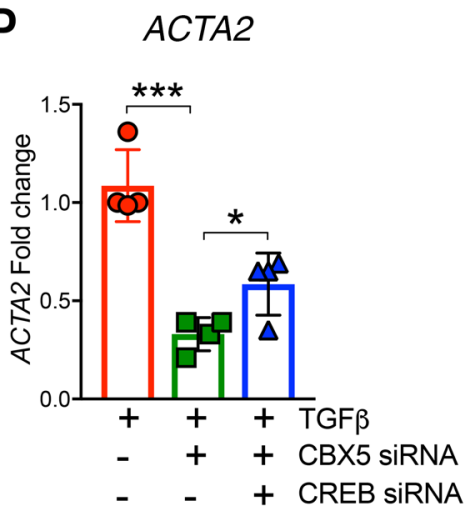

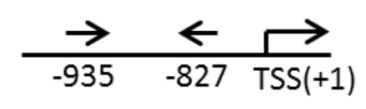
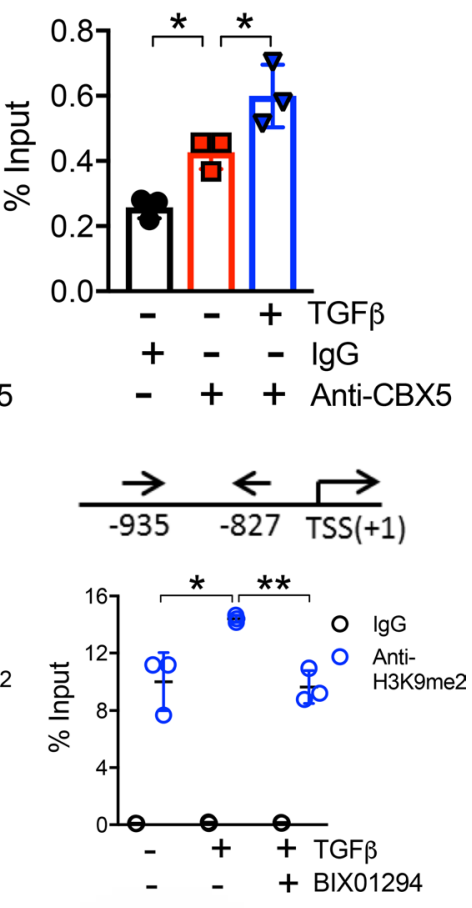

$\mathbf{E}$

E Transcriptionally inactive chromatin
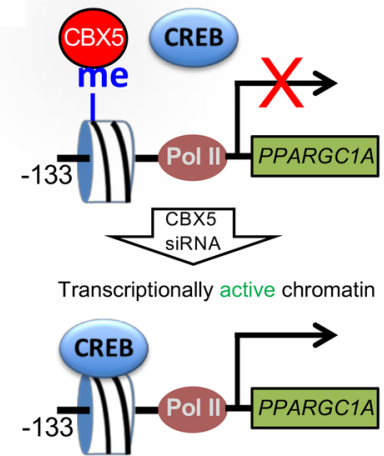

Figure 7. TGF- $\beta$ enhances CBX5 recruitment and H3K9me mark enrichment at the PPARGC1A gene promoter. (A) ChIP analysis showing CBX5 enrichment at the proximal promoter of PPARGC1A gene in human lung fibroblasts cultured on stiff plastic substrates. TGF- $\beta$ further elevates CBX5 recruitment at the PPARGC1A gene promoter $(n=3)$. Data are shown as mean $\pm \operatorname{SEM}\left({ }^{*} P<0.05,{ }^{* *} P<0.01,{ }^{* * *} P<0.001\right.$ by 2 -tailed, paired $t$ test). (B) ChIP analysis shows increased H3K9me2 mark decoration at the PPARGC1A gene promoter in human lung fibroblasts in response to TCF- $\beta$. BIX01294 treatment significantly reduces H3K 9 dimethylation at the PPARGC1A gene promoter in response to TCF- $\beta$. Three adjacent promoter regions were selected based on their affinity for transcription factors; starting from the transcription starting site (TSS) are CREB1, MEF2, and Fox01, respectively $(n=3)$. Data are shown as mean \pm SEM $(* P<0.05$ by 2-tailed, paired $t$ test) (C) qPCR analysis showing that inhibition of the transcription factor CREB by siRNA in CBX5-silenced fibroblasts reduces PPARGC1A gene expression. Data are shown as mean \pm SEM of 3 independent experiments performed in duplicate ( ${ }^{*} P<0.05$ by 1-way ANOVA with Turkey's multiple comparisons test). (D) CREB1 knockdown in lung fibroblasts reduces the beneficial effects of inhibiting CBX5 in TCF- $\beta$-treated fibroblasts. Data are shown as mean \pm SEM of 4 independent experiments performed in duplicate $\left({ }^{*} P<0.05,{ }^{* *} P<0.001\right.$ by 1 -way ANOVA with Turkey's multiple comparisons test). (E) Schematic representation of the proposed mechanism by which CBX5 binding to PPARGC1A gene promoter masks the CREB1-binding site and inhibits PPARGC1A gene transcription.

Ppargcla gene expression. As shown in Figure 8B, Ppargcla gene was strongly repressed at day 10 following bleomycin and its expression partially spontaneously recovered by day 20 after bleomycin. Interestingly, BIX01294 treatment at day 10 significantly elevated Ppargcla gene expression in whole lung at day 20 compared with vehicle control, thereby providing an important tool to manipulate Ppargcla gene expression during the progression of lung fibrosis. Gene expression analysis of disease-relevant genes also showed 
that expression of both $F n 1$ and $C T G F$ was significantly elevated in bleomycin-treated lungs compared with sham lungs, and BIX01294 treatment significantly attenuated their expression compared with vehicle (Figure 8, C and D). Histological examination revealed profound fibrotic remodeling in bleomycin-treated mice and a trend toward improved tissue architecture in animals that received a single dose of BIX01294 (Figure 8, E and F), though this effect did not reach statistical significance. Consistent with these observations, bleomycin-treated lungs showed a significant increase in hydroxyproline content, which was partially but not significantly reduced in the lungs of BIX01294-treated mice (Figure 8G). These latest observations, while failing to reach statistical significance, are supported by recent papers reporting the beneficial effects of BIX01294 in mouse models of kidney and peritoneal fibrosis $(60,61)$.

To directly investigate the contribution of G9a deletion in fibroblast activation during lung fibrosis, we used a conditional strategy to target G9a specifically in fibroblasts by generating mice with tamoxifen-inducible deletion in COL1 $\alpha 2$-expressing cells. To do this we crossed $g 9 a$-floxed mice $\left(g 9 a^{\mathrm{f} / \mathrm{fl}}\right)$ with transgenic mice expressing a tamoxifen-dependent Cre-ER(T) fusion protein under the control of the promoter for procollagen1 2 gene [Col1a2-CreER(T)]. To evaluate the fibrogenic response of the lungs, 5 doses of tamoxifen (once daily) were administered in these transgenic animals starting at day 3 after bleomycin administration (Figure $8 \mathrm{H}$ ). Immunofluorescence analysis of bleomycin-treated lungs harvested at day 21 showed absence of nuclear G9a and reduced H3K9me2 in PDGFR $\alpha$-expressing cells, demonstrating successful deletion of g9a gene in lung fibroblasts (Figure 8, I and K). Histological examination revealed significant fibrosis in bleomycin-treated Colla2-CreER(T): $g 9 a^{\mathrm{fl} / \mathrm{ll}}$ mice that did not receive tamoxifen and in bleomycin-treated controls $\left[g 9 a^{\mathrm{fl} / \mathrm{fl}}\right.$ and Colla2-CreER(T)] that received tamoxifen compared with sham animals. No significant changes, however, were observed in the lung architecture of bleomycin-treated Colla2-CreER(T): $g 9 a^{\mathrm{f} / \mathrm{fl}}$ mice treated with tamoxifen compared with sham animals (Figure 8, J and L). Additionally, hydroxyproline content was significantly elevated in the lungs of control mice [bleomycin-treated Colla2-CreER(T): $g 9 a^{\mathrm{f} / \mathrm{ft}}$ mice that did not receive tamoxifen as well as tamoxifen-treated Colla2-CreER(T) and $g 9 a^{\mathrm{f} / \mathrm{fl}}$ controls] but not in those with fibroblast-specific conditional deletion of $g 9 a$ [Colla2-CreER(T): $g 9 a^{\mathrm{f} / \mathrm{fl}}$ mice treated with tamoxifen] (Figure 8M). These observations demonstrate that G9a plays direct roles in lung fibroblast activation in vivo and is essential to pathological collagen deposition.

An important comparison here is between the efficacy of the global G9a inhibitor treatment and the statistically superior fibroblast-targeted deletion of G9a. While these experiments differ in the approach (pharmacologic and genetic) and time course, they suggest that nonfibroblast effects of G9a inhibition may complicate its net in vivo efficacy. Taken together, our findings thus demonstrate an important role for G9a inhibition in restoring Ppargcla gene expression in fibrotic lungs and in attenuating the activated fibroblast phenotype. Moreover, these results shed light on the potentially novel concept that promoting gene transcription through targeted epigenetic intervention may represent an appealing strategy aimed at restoring the altered transcriptional imbalance that promotes lung fibrosis progression.

\section{Discussion}

In this work we demonstrate that $\mathrm{CBX} 5 / \mathrm{G} 9 \mathrm{a} / \mathrm{H} 3 \mathrm{~K} 9$ me-mediated gene repression is critical to initiate and perpetuate lung fibroblast activation in response to both biochemical and biomechanical stimuli. Mechanistically, we show that this pathway epigenetically represses PPARGC1A gene and PGC1 $\alpha$ protein expression and that this repression is required for sustained fibrogenic activation (Figure 9). Gene expression analysis of FACS-sorted mouse fibroblasts isolated from the lungs of bleomycin-treated animals showed that Ppargcla gene expression was markedly decreased in vivo during fibrosis, while immunofluorescence imaging from both diseased mouse and human IPF lungs confirmed strong H3K9me in areas of active fibrogenesis. Diseased lung fibroblasts isolated from bleomycin-treated mice displayed stably reduced PGC1 $\alpha$ expression and elevated ECM protein deposition, even when cultured in vitro, demonstrating a "fibrogenic memory" in vitro that appears dependent on epigenetic gene repression to sustain their activated state. Interestingly, we demonstrated elevation of CBX5 expression in IPF-derived compared with normal fibroblasts, suggesting a potential role for CBX5 in perpetuating fibroblast activation ex vivo. Most importantly, inhibition of the G9a/CBX5 pathway in diseased mouse or human lung fibroblasts restored PGC1 $\alpha$ expression and strongly attenuated their fibrogenic activation. Additionally, global inhibition of G9a in vivo by BIX01294 elevated Ppargcla gene expression and partially attenuated lung fibrosis in mice challenged with bleomycin compared with control animals, suggesting that targeting this epigenetic repressive mechanism may have therapeutic benefits. 
A
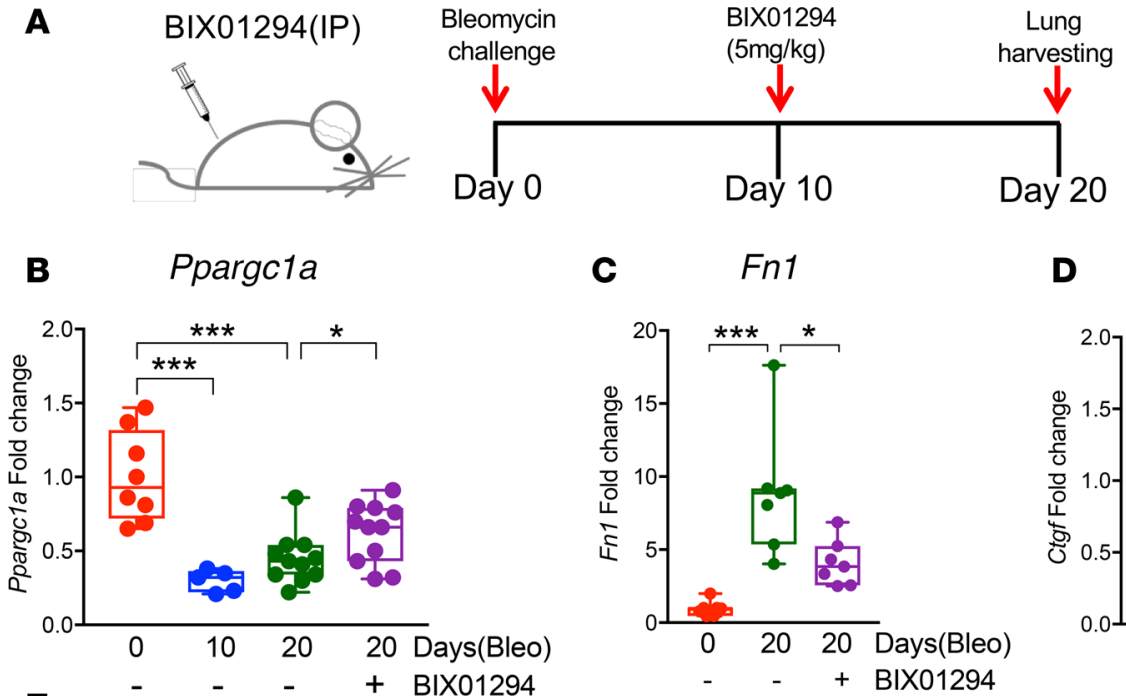

D Ctgf

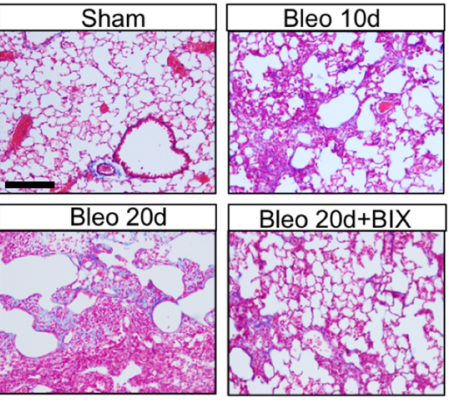

F

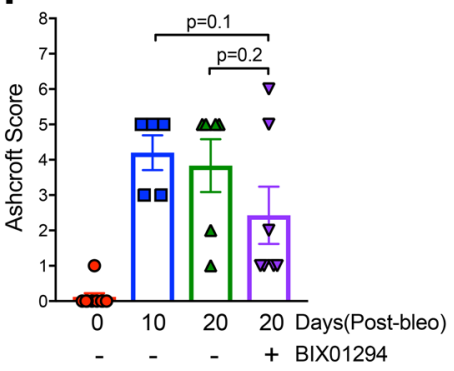

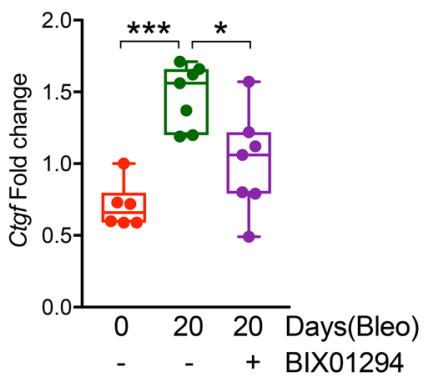

G

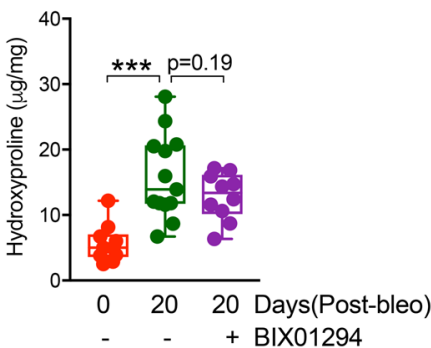

H

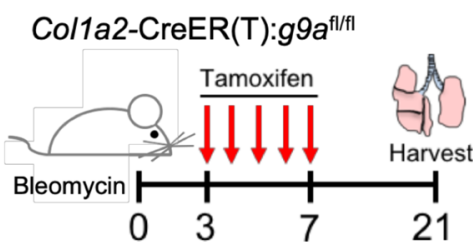

J

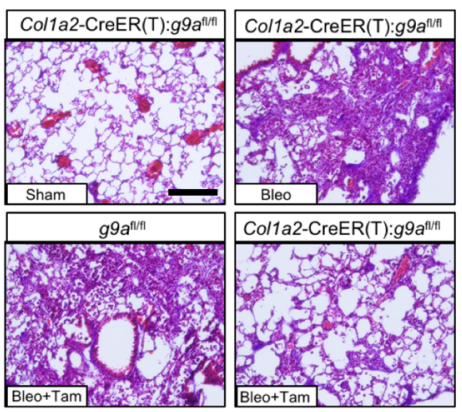

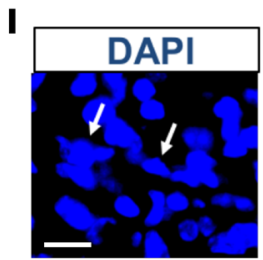

K

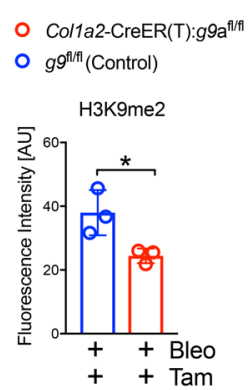

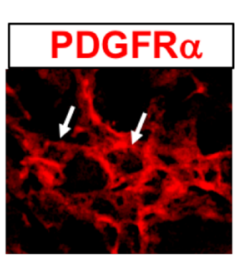

L

O Col1a2-CreER(T):g9a $a^{\text {n/f }}$ - Col1a2-CreER(T) (Control) $g^{9 / / 1 /}$ (Control)

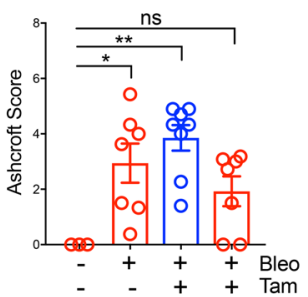

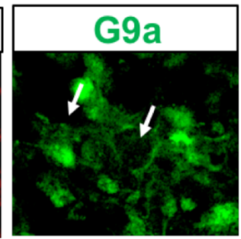

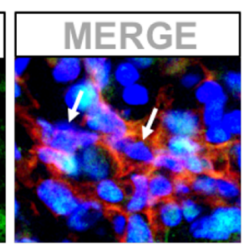

M

O Col1a2-CreER(T): $g 9 a^{\mathrm{f} / / \mathrm{t}}$

- Col1a2-CreER(T) (Control) $g^{f f / f l}$ (Control)

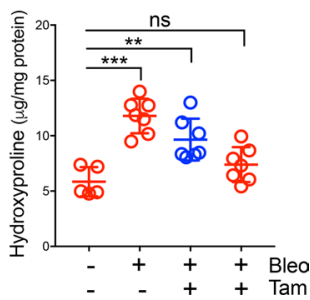

Figure 8. Inhibition of G9a following bleomycin challenge promotes Ppargc1a gene expression and attenuates lung fibrosis. (A) Schematic showing experimental workflow. (B) qPCR showing Ppargc1a gene repression following bleomycin delivery. One single dose of BIX01294 partially restores Ppargc1a gene expression compared with vehicle-treated animals $(n=5)$. Data are shown as mean $\pm \mathrm{SEM}\left({ }^{*} P<0.05,{ }^{* * *} P<0.001\right.$ by 1 -way ANOVA with Turkey's multiple comparisons test). (C and D) qPCR shows reduced Fn1 and Ctgf transcripts in BIX01294-treated mice lungs compared with vehicle-treated animals $(n=6)$. Data are shown as mean \pm SEM $\left({ }^{*} P<0.05\right.$, ${ }^{* *} P<0.001$ by 1 -way ANOVA with Turkey's multiple comparisons test). (E) Representative images of Masson's trichome-stained lung tissues. Scale bar: $200 \mu \mathrm{m}$. (F) H\&E-stained lung sections were scored using Ashcroft method. (G) Hydroxyproline assay was used to evaluate collagen deposition in the lungs. Sham control $(n=8)$, Bleo 20 days control ( $n=12$ ), and Bleo 20 days BIX01294 $(n=11)$. Data are shown as mean \pm SEM (*** $P<0.001$ by 1 -way ANOVA with Turkey's multiple comparisons test). (H) Strategy for induc-

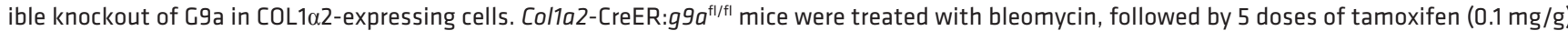
starting at day 3. (I) Immunofluorescence images show G9a deletion in PDCFR $\alpha^{+}$cells. Arrows indicate cell nuclei that are negative for G9a staining. Scale bar: $10 \mu \mathrm{m}$. (J) Representative images of Masson's trichome-stained lung tissues. Scale bar: $200 \mu \mathrm{m}$. (K) Reduced H3K9me2 in fibroblasts from tamoxifen-treated Col1a2-CreER(T): $g 9 a^{\mathrm{fl} / \mathrm{fl}}$ mice compared with $g 9 a^{\mathrm{fl} / \mathrm{fl}}$ control mice. (L) H\&E-stained lung sections were scored using Ashcroft method. 
(M) Bleomycin significantly increases hydroxyproline content in the lungs of control mice [Col1a2-CreER(T):g9a $a^{\text {fl/fl }}$ mice not treated with tamoxifen ( $n$ $=7)$ as well as $g 9 a^{f / / f l}$ and Col7a2-CreER(T) control groups treated with tamoxifen $(n=7)$ ] but not in mice with conditional deletion of C9a in fibroblasts $\left[\right.$ Col1a2-CreER $(\mathrm{T}): g 9 a^{\mathrm{fl} / \mathrm{fl}}$ group treated with tamoxifen, $\left.n=7\right]$ compared with uninjured controls $(n=5)$. Data are shown as mean $\pm \mathrm{SEM}\left({ }^{* *} P<0.01\right.$, ${ }^{* * *} P<0.001$ by 1 -way ANOVA with Turkey's multiple comparisons test).

PGC1 $\alpha$ is the coactivator of the peroxisomal family member PPAR $\gamma$ and is well known for its critical role during mitochondria biogenesis and fatty acid metabolism $(29,30)$. Interestingly, both mitochondrial dysfunction and deficient fatty acid metabolism have been shown to contribute to the development of fibrosis in multiple organs $(31,62,63)$. In this regard, it has recently been shown that loss of PGC1 $\alpha$ in lung alveolar epithelial cells leads to multiple mitochondrial perturbations, including deficient oxidative respiration and misregulated apoptosis, which directly contributes to the development of interstitial fibrogenesis (64). Additionally, a recent paper revealed that renal tubular epithelial cells critically depend on fatty acid oxidation as their energy source, and reduced PGC1 $\alpha$ expression has been observed in epithelial cells of the kidneys of patients with chronic kidney disease, supporting a protective role for PGC1 $\alpha$ in the setting of chronic diseases (62). Although these recent publications demonstrate that the loss of PGC1 $\alpha$ in epithelial cells contributes to the development of kidney and lung fibrosis, the role of PGC1 $\alpha$ in fibroblast activation, its loss in disease settings, and the mechanisms responsible for its gene repression have not been previously studied. Our work identifies an inhibitory epigenetic mechanism responsible for the transcriptional repression of PGC1 $\alpha$ in diseased lung fibroblasts and reveals, for the first time to our knowledge, that PGC1 $\alpha$ is essential to restrain fibroblast activation, thereby identifying an epigenetic foundation that underpins the switch between lung fibroblast quiescence and activation through repression of a fibrosis suppressor gene.

In the context of TGF- $\beta$ signaling, our findings demonstrate that PGC1 $\alpha$ is a direct target of a repressive program initiated through the engagement of the G9a/CBX5 epigenetic pathway and that the repression of PGC1 $\alpha$ is essential to fibroblast activation by TGF- $\beta$. Interestingly, even in the absence of TGF- $\beta$, BIX01294 elevated PPARGC1A gene expression in IPF-derived fibroblasts and this elevation preceded the attenuation of ACTA2 gene expression, further suggesting that G9a-mediated PPARGC1A gene repression is essential to the persistent fibrogenic activation of these cells. Other epigenetic transcriptional repressors, such as HDACs and DNA methyltransferases (DNMT1), have been shown to play a role in TGF- $\beta$ signaling and contribute to sustained fibrogenesis $(11,21,65,66)$. Intriguingly, multiple HDACs, including HDAC5 and HDAC9 as well as DNMT1, have been shown to directly interact with CBX5 to maximize their repressive functions $(35,49)$, suggesting that CBX5 might orchestrate a platform of multiple epigenetic repressors to ensure stable silencing of fibrosis suppressor genes.

In addition to exerting its repressive function on the PPARGC1A gene, we found that TGF- $\beta$ potently repressed $P P A R A$ and $P P A R G, 2$ genes that are closely related to, and transcriptionally regulate, PPARGC1A (67), suggesting that repression of these genes by TGF- $\beta$ may diminish PPARGC1A gene transcription. Numerous studies have identified important roles of PPAR $\alpha$ and PPAR $\gamma$ in regulating processes related to fibrogenesis, including cellular differentiation, inflammation, and wound healing $(54,56)$. PPAR $\alpha$ agonists inhibit the production of collagen by hepatic stellate cells and inhibit liver, kidney, and cardiac fibrosis in animal models (68-70). In the bleomycin model of lung fibrosis PPARo agonists significantly inhibit the fibrotic response, while PPAR $\alpha$-deficient mice developed more serious fibrosis (71). Interestingly, while we confirmed that inhibition of G9a by BIX01294 blocked TGF- $\beta$ induced PPARGC1A gene repression at 4 hours and significantly blocked TGF- $\beta$ profibrotic responses within 12 hours, we did not observed PPARA or PPARG restoration by BIX01294, demonstrating that these genes are not directly responsible for the antifibrotic effect of BIX01294. Similar to what we found using the G9a inhibitor BIX01294, only inhibition of PGC1 $\alpha$ fully reversed the CBX5 siRNAmediated inhibition of ACTA2 gene expression, confirming the leading role of PGC1 $\alpha$ as a fibrosis suppressor in our studies. Intriguingly, previous studies on liver fibrosis have demonstrated a direct contribution for MeCP2 and EZH2, two epigenetic repressors involved in H3K9 and H3K27 methylation, respectively, and CBX5 binding $(25,35)$, in TGF- $\beta$-mediated PPAR $\gamma$ repression and hepatic stellate cell activation (72). Similarly, while our findings support a direct role for CBX5 in promoting TGF- $\beta$-induced PPARG gene repression in lung fibroblasts, only CBX5 but not G9a inhibition in these cells rescued PPARG gene expression, suggesting that different epigenetic dynamics may control peroxisomal gene repression in lung and liver mesenchymal cells to promote their activation. 


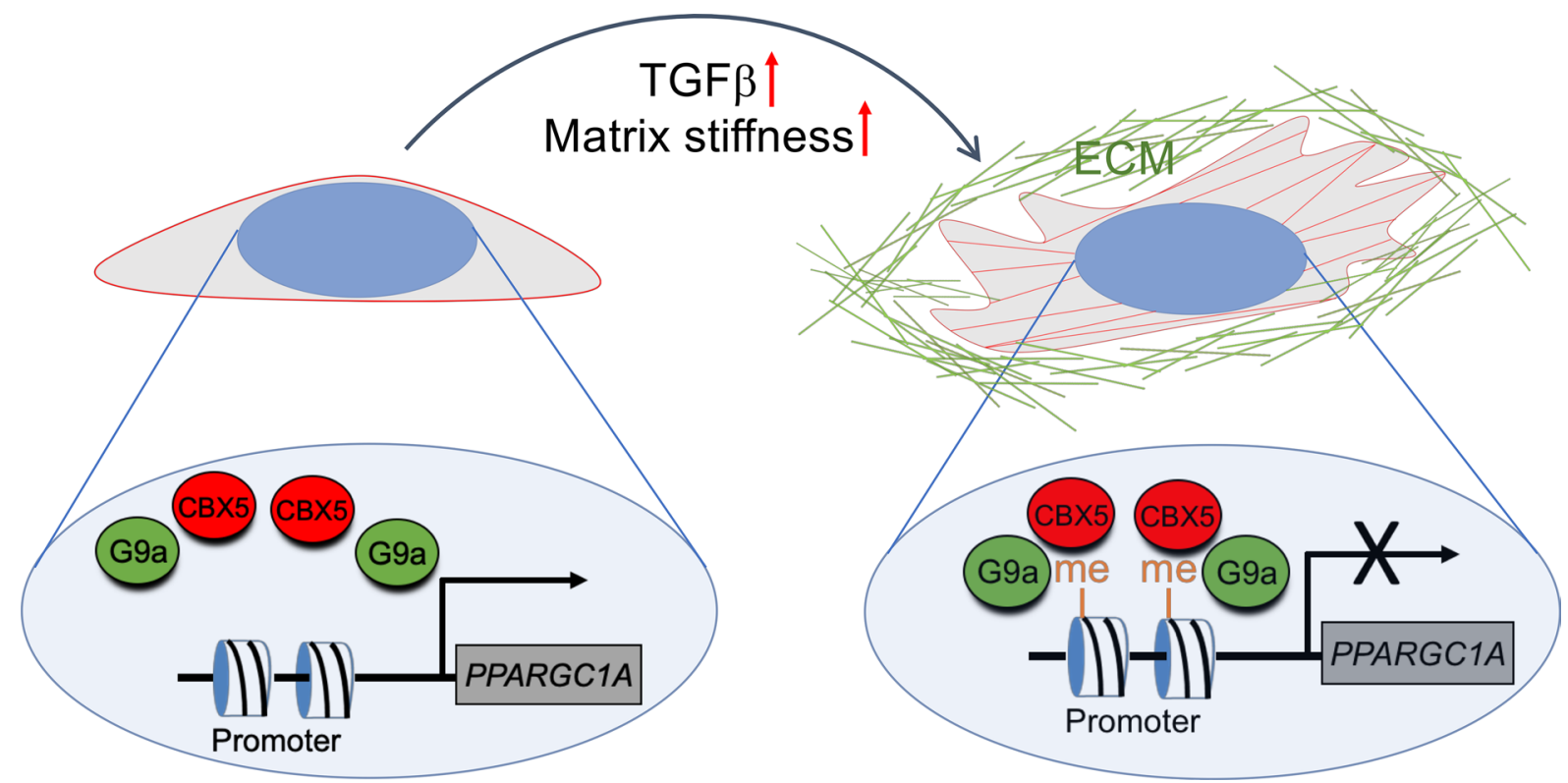

Figure 9. Schematic representation of the mechanism leading to CBX5/G9a/H3K9me-mediated PPARGC1A gene repression and lung fibroblast activation in response to TGF- $\beta$ and matrix stiffness.

In addition to their responsiveness to TGF- $\beta$, lung fibroblasts are exquisitely sensitive to the mechanical milieu of the lung (42). In fibrotic scar tissue, the mechanical environment is profoundly altered and increases in the tissue stiffness directly contribute to fibroblast activation (73). Although previous studies have shown that mechanical cues and matrix stiffness can alter nuclear functions, including chromatin organization (73), no prior studies have addressed the role of histone modification, especially H3K9 methylation, during fibroblast activation as a result of the altered lung mechanical microenvironment. Our work demonstrates that lung fibroblasts cultured on pathologically stiff substrates are characterized by elevated global H3K9 methylation compared with those cultured on physiologically compliant substrates. Inhibition of G9a or CBX5 strongly inhibited matrix stiffness-induced global H3K9 methylation and reduced $\alpha \mathrm{SMA}$ expression and traction forces, thereby confirming the relevance of H3K9 methylation during fibroblast mechanoactivation. Interestingly, our findings are in line with those in a recent paper showing that in cancer cells mechanical signals generated from rigid substrates promote epigenetic remodeling through $\mathrm{H} 3 \mathrm{~K} 9$ methylation-mediated gene repression (74). Intriguingly, inhibition of Cdc42, a critical regulator of cytoskeletal assembly, reduced H3K9 methylation in cancer cells that were in contact with stiff substrates. These observations, together with our data, suggest that tension-dependent cytoskeletal mechanotransduction may alter chromatin organization to promote repressive mark deposition in genomic regions containing tumoror fibrosis-suppressor genes. In line with these observations, immunostaining of histological sections of bleomycin-treated lungs as well as lung tissue from IPF patients, revealed elevated global H3K9 methylation in fibrotic region of the lung that displayed abundant ECM deposition, suggesting that the in vivo environment of the lung may directly influence chromatin state. While earlier studies have shown that nucleus-cytoskeleton interconnectivity regulates genome-wide transcriptional responses to substrate rigidity (73), the epigenetic mechanisms underlining these responses have not been fully investigated. Our work partially fills this gap and demonstrates that the G9a/CBX5 epigenetic pathway plays a critical role in the mechanoresponses of lung fibroblasts by translating mechanical signals into chromatin alterations that promote gene repression in support of fibroblast activation. Although, in this paper, we did not directly investigate the mechanosignaling pathway leading to the increased H3K9 methylation, we speculate that the profound cytoskeleton alterations that take place in fibroblasts interacting with a pathological stiff environment may directly influence nuclear dynamics and chromatin organization, as supported by recent studies reporting G9a and CBX5 interactions with the nuclear envelope $(75,76)$. Together our observations shed light on the role of the G9a/ $\mathrm{CBX} 5 / \mathrm{H} 3 \mathrm{~K} 9$ me epigenetic pathway during fibroblast activation in response to mechanical and biochemical cues and set the stage for future investigations aimed at understanding how these signals alter nuclear and chromatin dynamics to orchestrate gene repression and promote fibroblast activation. 
Finally, given that epigenetic alterations are transmissible and can perpetuate the altered state of the cell through cell division, our findings provide a strong rationale for targeting epigenetically repressed genes such as PPARGC1A in diseased lung fibroblast to reestablish their quiescence and halt progressive lung fibrogenesis. In this regard, G9a inhibition by BIX01294 in mice that have been challenged with bleomycin strongly reduced global H3K9 methylation in whole lung, elevated PPARGC1A gene expression and showed a trend toward beneficial effect in limiting fibrosis progression. Cell-specific genetic targeting of G9a in fibroblasts was even more effective in reducing experimental fibrosis. Although drugs that target epigenetic regulators have been approved for the treatment of cancer, this type of therapeutic intervention has not been advanced to treat fibrogenic disorders. Our data underline the importance of exploring epigenetic gene repression as a potentially targetable mechanism to reverse the pathological activated state of lung fibroblasts and more broadly halt lung fibrosis progression.

\section{Methods}

Cell culture. Cells were maintained in EMEM (ATCC) containing 10\% FBS, 1\% antibiotics. IMR-90 embryonic lung fibroblasts were purchased from ATCC. Primary human lung fibroblasts (HLF) isolated by explant culture from the lungs of subjects diagnosed with IPF who underwent lung transplantation or donors (HLF) whose organs were rejected for transplantation (non-IPF) were provided by Peter Bitterman and Craig Henke at the University of Minnesota (Minneapolis, MN) under a protocol approved by the University of Minnesota Institutional Review Board. All primary cell culture experiments were performed with cells at passage 6 or less.

Reagents. BIX01294 (catalog B9311) and (+)-JQ1 (catalog SML1524) were from MilliporeSigma.

Human samples. Healthy lung samples were from surgical resections of patients undergoing thoracic surgery at St. Mary's Hospital, Mayo Clinic Rochester, for focal pathologies (e.g., primary lung cancers without metastases; bronchoalveolar carcinoma and infectious pathologies excluded), and IPF lung samples were obtained from patients who underwent lung transplantation.

Mice. Col1 1 1-GFP-transgenic mice were generated as previously described (University of California, San Diego, La Jolla, California, USA) (77) and provided by Derek Radisky (Mayo Clinic, Jacksonville, FL). Conditional COL1 $\alpha 2$ cell-specific $g 9 a\left[\right.$ Colla2-CreER(T)- $\left.g 9 a^{\mathrm{f} / \mathrm{fl}}\right]$ mice were generated by breeding of Col1a2-CreER(T) mice (Jackson Laboratory) with $g 9 a^{\mathrm{f} / \mathrm{fl}}$ mice. Eight-week-old female and male C57/BL6 mice were purchased from Jackson Laboratory.

$R N A$ interference. RNA interference was performed using Lipofectamine RNAiMAX reagent (Thermo Fisher Scientific) as described in the Supplemental Methods.

Immunofluorescence staining. Mouse and human tissues were immunostained by using Fibronectin I (Santa Cruz, sc-9068), H3K9me2 (Cell signaling, 4658S), aSMA (MilliporeSigma, F3777), and G9a (Abcam, ab40542) primary antibodies, as described in the Supplemental Methods.

Fibrosis evaluation. Seven- $\mu \mathrm{m}$-thick sections were cut from paraffin-embedded lung tissues, and the sections were stained either with H\&E or with Masson's trichrome stain kit (Abcam). All H\&E-stained slides and trichrome-stained slides were reviewed in a blinded fashion by a thoracic pathologist. The severity of the fibrosis was evaluated using Ashcroft score: 0 (no fibrosis), 1 (minimal interstitial and/or peribronchiolar thickening due to fibrosis), 3 (moderate thickening without obvious architectural distortion), 5 (increased fibrosis with formation of fibrous bands and/or small masses), 7 (severe architectural distortion with large areas of fibrosis and areas of honeycomb changes), and 8 (total fibrous obliteration of the field) (78). The mean of all scores was calculated for each mouse. Hydroxyproline content was measured using a hydroxyproline colorimetric assay kit (Biovision), as described in the Supplemental Methods.

Traction force microscopy. Traction analysis was performed as previously described (32). Fluorescent sulfate-modified latex microspheres $(0.2 \mu \mathrm{m}, 505 / 515 \mathrm{ex} / \mathrm{em}$, FluoSpheres, Life Technologies) were conjugated to the gel surfaces of polyacrylamide substrates with shear moduli of $6.4 \mathrm{kPa}$ after treatment with $1 \mathrm{mg} /$ $\mathrm{ml}$ dopamine hydrochloride (MilliporeSigma) dissolved in $50 \mathrm{mM}$ HEPES solution ( $\mathrm{pH}$ 8.5). Cells were transfected with scramble or CBX5 or G9a siRNA and then transferred on the gel plates overnight before traction force measurements. Images of gel surface-conjugated fluorescent beads were acquired for each cell before and after trypsinization using a Nikon ECLIPSE Ti microscope at $\times 10$ magnification. Traction forces were evaluated by measuring bead displacement fields and computing corresponding traction fields using TractionsForAll (http://www.mayo.edu/research/labs/tissue-repair-mechanobiology/software).

Real-time PCR. Total mRNA was isolated and the relative gene expression was analyzed as described in the Supplemental Methods. Primers used for qPCR are listed in Supplemental Figure 4. 
Protein extraction and Western blotting analysis. Western blotting analysis of protein lysates was performed as described in the Supplemental Methods. Densitometry analysis of all Western blots is shown in Supplemental Figure 3.

Wound-healing assay. Cell migration was evaluated using wound-healing assay. After scramble or CBX5 siRNA transfection of cells in 24-well plates, a single scratch was made with sterile 200 - $\mu$ l pipette tip to obtain a cell-free area, followed by washing of cell monolayer with serum-free medium and treatment with TGF- $\beta$ ( 2 ng/ml). Cells were photographed at 12 and 24 hours using Olympus CKX53 inverted microscope equipped with a digital camera. The wound area was measured using ImageJ (NIH) software.

FACS sorting. Single cells were sorted from mice lungs after bleomycin or vehicle delivery as described in the Supplemental Methods.

Mouse model of bleomycin-induced lung injury. Mouse lung fibrosis was induced with bleomycin (Fresenius Kabi) delivered intratracheally $(1.1 \mathrm{U} / \mathrm{kg}$ ) to the lungs using MicroSprayer Aerosolizer (Penn-Century) as previously described (32). The sham mice received sterile $0.9 \%$ saline instead using identical methods. Body weight was monitored daily. For the BIX01294 study, mice were weighed every 24 hours, and both groups were then randomized at day 10 into BIX01294 and control treatment groups. One single dose of BIX01294 (5 mg/kg) was intraperitoneally delivered at day 10 following bleomycin administration. The control groups of mice received the equivalent vehicle dose of PBS. Ten days following BIX01294 treatment, mice were sacrificed and the right lungs were inflated with $4 \%$ paraformaldehyde (PFA) and further incubated in 4\% PFA for 24 hours prior processing for paraffin embedding. The left lobe of the lung was snap frozen in liquid nitrogen for RNA isolation and hydroxyproline assay. For experiments with the colla2-creER(T)- $g 9 a^{\mathrm{f} / \mathrm{fl}}$-transgenic mice tamoxifen (MilliporeSigma) dissolved in corn oil was injected i.p. at 5 doses of $0.1 \mathrm{mg} / \mathrm{g}$ body weight to induce Cre-mediated recombination.

ECM deposition assay. Cellular ECM deposition was measured using primary Collagen I (Novus, NB600408) and Fibronectin (MilliporeSigma, sc-9068) antibodies as described in the Supplemental Methods.

Collagen gel contraction assay. After scramble or CBX5 or G9a siRNA transfection (48 hours), cells were mixed into collagen solution $(2 \mathrm{mg} / \mathrm{ml})$ in presence of TGF- $\beta(2 \mathrm{ng} / \mathrm{ml})$. Collagen/cell suspensions were incubated at $37^{\circ} \mathrm{C}$ for 1 hour to allow the collagen gels to solidify. Gels were then gently lifted from the wells and the diameter of each gel was measured using an inverted microscope.

ChIP. ChIP assay was performed following the manufacturer's instructions (ChIP Assay Kit, MilliporeSigma). Cells were subjected to cross-linking with $1 \%$ formaldehyde in medium for 10 minutes at $37^{\circ} \mathrm{C}$ and then lysed in SDS buffer for 10 minutes on ice. Chromatin was sonicated to shear DNA to an average length of $0.2-1.0 \mathrm{~kb}$. Antibodies against CBX5 (Cell Signaling, 2616S) and H3K9me2 (Cell Signaling, 4658P) were used for immunoprecipitation. Normal mouse IgG (Santa Cruz) was used as negative control. The immunoprecipitation was heated to reverse the formaldehyde cross-linking, and the DNA fragments in the precipitates were purified by phenol/chloroform extraction and ethanol precipitation. The immunoprecipitated DNA was quantified by real-time PCR. Primers were designed to amplify the proximal promoter region of PPARGC1A gene. Primers are listed in Supplement Figure 4.

Statistics. Data are presented as the mean \pm SEM. The comparison between 2 groups was calculated with Student's paired, 2-tailed $t$ test, and group analysis was compared by using 1-way ANOVA with Tukey's multiple comparison's test using GraphPad Prism 6.0, with statistical significance defined as $P<0.05$. Results are expressed throughout with box-and-whisker plots showing minimum to maximum, quartile, and median.

Study approval. All animal experiments were carried out under protocols approved by the Mayo Clinic Institutional Animal Care and Use Committee, and the animals were handled in accordance to their guidelines. Informed consent was obtained from subjects or their legal guardians during initial medical workup.

\section{Author contributions}

GL and DJT conceived and designed the project. GL, NC, JAM, DLJ, QT, KMC, AJH, and AA performed the experiments. GL, YSP, RAU, GL, and DJT interpreted the data. GL and DJT wrote the manuscript. ACR reviewed mouse tissue slides. All authors edited and approved the manuscript.

\section{Acknowledgments}

Funding support was provided by NIH grants HL142596 (to GL), HL092961 and HL133320 (to DJT). 
Address correspondence to: Daniel J. Tschumperlin or Giovanni Ligresti, Mayo Clinic College of Medicine and Science, 200 First Street SW, Rochester, Minnesota 55905, USA. Phone: 507.255.8475; Email: tschumperlin.daniel@mayo.edu (D.J. Tschumperlin). Phone: 617.358.6786; Email: ligresti.giovanni@mayo. edu or ligresti@bu.edu (G. Ligresti).

GL's present address is: Department of Medicine, Boston University School of Medicine, Boston, Massachusetts, USA.

1. Hinz B, Phan SH, Thannickal VJ, Galli A, Bochaton-Piallat ML, Gabbiani G. The myofibroblast: one function, multiple origins. Am J Pathol. 2007;170(6):1807-1816.

2. Barkauskas CE, Noble PW. Cellular mechanisms of tissue fibrosis. 7. New insights into the cellular mechanisms of pulmonary fibrosis. Am J Physiol, Cell Physiol. 2014;306(11):C987-C996.

3. Kendall RT, Feghali-Bostwick CA. Fibroblasts in fibrosis: novel roles and mediators. Front Pharmacol. 2014;5:123.

4. Scotton CJ, Chambers RC. Molecular targets in pulmonary fibrosis: the myofibroblast in focus. Chest. 2007;132(4):1311-1321.

5. Bochaton-Piallat ML, Gabbiani G, Hinz B. The myofibroblast in wound healing and fibrosis: answered and unanswered questions. F1000Res. 2016;5:F1000 Faculty Rev-752.

6. Tschumperlin DJ. Matrix, mesenchyme, and mechanotransduction. Ann Am Thorac Soc. 2015;12 Suppl 1:S24-S29.

7. Liu F, et al. Feedback amplification of fibrosis through matrix stiffening and COX-2 suppression. J Cell Biol. 2010;190(4):693-706.

8. Wells RG. The role of matrix stiffness in regulating cell behavior. Hepatology. 2008;47(4):1394-1400.

9. Yao HW, Li J. Epigenetic modifications in fibrotic diseases: implications for pathogenesis and pharmacological targets. J Pharmacol Exp Ther. 2015;352(1):2-13.

10. Helling BA, Yang IV. Epigenetics in lung fibrosis: from pathobiology to treatment perspective. Curr Opin Pulm Med. 2015;21(5):454-462.

11. Tzouvelekis A, Kaminski N. Epigenetics in idiopathic pulmonary fibrosis. Biochem Cell Biol. 2015;93(2):159-170.

12. Perugorria MJ, et al. Histone methyltransferase ASH1 orchestrates fibrogenic gene transcription during myofibroblast transdifferentiation. Hepatology. 2012;56(3):1129-1139.

13. Rennert O, Lee TL. Epigenetics dynamics in development and disease. Int J Biochem Cell Biol. 2015;67:44.

14. Vogel T, Lassmann S. Epigenetics: development, dynamics and disease. Cell Tissue Res. 2014;356(3):451-455.

15. Hirst M, Marra MA. Epigenetics and human disease. Int J Biochem Cell Biol. 2009;41(1):136-146.

16. Rabinovich EI, Selman M, Kaminski N. Epigenomics of idiopathic pulmonary fibrosis: evaluating the first steps. Am J Respir Crit Care Med. 2012;186(6):473-475.

17. Tang X, et al. Assessment of Brd4 inhibition in idiopathic pulmonary fibrosis lung fibroblasts and in vivo models of lung fibrosis. Am J Pathol. 2013;183(2):470-479.

18. Tang $\mathrm{X}$, et al. BET bromodomain proteins mediate downstream signaling events following growth factor stimulation in human lung fibroblasts and are involved in bleomycin-induced pulmonary fibrosis. Mol Pharmacol. 2013;83(1):283-293.

19. Coward WR, Brand OJ, Pasini A, Jenkins G, Knox AJ, Pang L. Interplay between EZH2 and G9a Regulates CXCL10 Gene Repression in Idiopathic Pulmonary Fibrosis. Am J Respir Cell Mol Biol. 2018;58(4):449-460.

20. Huang SK, et al. Hypermethylation of PTGER2 confers prostaglandin E2 resistance in fibrotic fibroblasts from humans and mice. Am J Pathol. 2010;177(5):2245-2255.

21. Coward WR, Watts K, Feghali-Bostwick CA, Jenkins G, Pang L. Repression of IP-10 by interactions between histone deacetylation and hypermethylation in idiopathic pulmonary fibrosis. Mol Cell Biol. 2010;30(12):2874-2886.

22. Coward WR, Feghali-Bostwick CA, Jenkins G, Knox AJ, Pang L. A central role for G9a and EZH2 in the epigenetic silencing of cyclooxygenase-2 in idiopathic pulmonary fibrosis. FASEB J. 2014;28(7):3183-3196.

23. Cisneros J, et al. Hypermethylation-mediated silencing of p14(ARF) in fibroblasts from idiopathic pulmonary fibrosis. $A m \mathrm{~J}$ Physiol Lung Cell Mol Physiol. 2012;303(4):L295-L303.

24. Fischle W, et al. Regulation of HP1-chromatin binding by histone H3 methylation and phosphorylation. Nature. 2005;438(7071):1116-1122.

25. Stewart MD, Li J, Wong J. Relationship between histone H3 lysine 9 methylation, transcription repression, and heterochromatin protein 1 recruitment. Mol Cell Biol. 2005;25(7):2525-2538.

26. Eissenberg JC, Elgin SC. HP1a: a structural chromosomal protein regulating transcription. Trends Genet. 2014;30(3):103-110.

27. Krishnan S, Horowitz S, Trievel RC. Structure and function of histone H3 lysine 9 methyltransferases and demethylases. Chembiochem. 2011;12(2):254-263.

28. Shinkai Y, Tachibana M. H3K9 methyltransferase G9a and the related molecule GLP. Genes Dev. 2011;25(8):781-788.

29. Liang H, Ward WF. PGC-1alpha: a key regulator of energy metabolism. Adv Physiol Educ. 2006;30(4):145-151.

30. Finck BN, Kelly DP. PGC-1 coactivators: inducible regulators of energy metabolism in health and disease. J Clin Invest. 2006;116(3):615-622.

31. Mora AL, Bueno M, Rojas M. Mitochondria in the spotlight of aging and idiopathic pulmonary fibrosis. J Clin Invest. 2017;127(2):405-414.

32. Oh RS, et al. RNAi screening identifies a mechanosensitive ROCK-JAK2-STAT3 network central to myofibroblast activation J Cell Sci. 2018;131(10):jcs209932.

33. Wang J, et al. Pharmacological targeting of BET proteins attenuates radiation-induced lung fibrosis. Sci Rep. 2018;8(1):998.

34. Hyun K, Jeon J, Park K, Kim J. Writing, erasing and reading histone lysine methylations. Exp Mol Med. 2017;49(4):e324.

35. Lan F, Shi Y. Epigenetic regulation: methylation of histone and non-histone proteins. Sci China, C, Life Sci. 2009;52(4):311-322.

36. Ding N, et al. BRD4 is a novel therapeutic target for liver fibrosis. Proc Natl Acad Sci USA. 2015;112(51):15713-15718.

37. Vogel ER, et al. Cigarette smoke enhances proliferation and extracellular matrix deposition by human fetal airway smooth mus- 
cle. Am J Physiol Lung Cell Mol Physiol. 2014;307(12):L978-L986.

38. Wilborn J, Crofford LJ, Burdick MD, Kunkel SL, Strieter RM, Peters-Golden M. Cultured lung fibroblasts isolated from patients with idiopathic pulmonary fibrosis have a diminished capacity to synthesize prostaglandin E2 and to express cyclooxygenase-2. J Clin Invest. 1995;95(4):1861-1868.

39. Moodley YP, et al. Fibroblasts isolated from normal lungs and those with idiopathic pulmonary fibrosis differ in interleukin-6/ gp130-mediated cell signaling and proliferation. Am J Pathol. 2003;163(1):345-354

40. Standiford TJ, et al. Altered production and regulation of monocyte chemoattractant protein-1 from pulmonary fibroblasts isolated from patients with idiopathic pulmonary fibrosis. Chest. 1993;103(2 Suppl):121S.

41. Tschumperlin DJ, Boudreault F, Liu F. Recent advances and new opportunities in lung mechanobiology. J Biomech. 2010;43(1):99-107.

42. Huang X, et al. Matrix stiffness-induced myofibroblast differentiation is mediated by intrinsic mechanotransduction. Am J Respir Cell Mol Biol. 2012;47(3):340-348.

43. Balestrini JL, Chaudhry S, Sarrazy V, Koehler A, Hinz B. The mechanical memory of lung myofibroblasts. Integr Biol (Camb). 2012;4(4):410-421.

44. Liu F, et al. Mechanosignaling through YAP and TAZ drives fibroblast activation and fibrosis. Am J Physiol Lung Cell Mol Physiol. 2015;308(4):L344-L357.

45. Fritsch L, et al. A subset of the histone H3 lysine 9 methyltransferases Suv39h1, G9a, GLP, and SETDB1 participate in a multimeric complex. Mol Cell. 2010;37(1):46-56.

46. Smallwood A, Estève PO, Pradhan S, Carey M. Functional cooperation between HP1 and DNMT1 mediates gene silencing. Genes Dev. 2007;21(10):1169-1178.

47. Leung DC, et al. Lysine methyltransferase G9a is required for de novo DNA methylation and the establishment, but not the maintenance, of proviral silencing. Proc Natl Acad Sci USA. 2011;108(14):5718-5723.

48. Sampath SC, et al. Methylation of a histone mimic within the histone methyltransferase G9a regulates protein complex assembly. Mol Cell. 2007;27(4):596-608.

49. Aygün O, Mehta S, Grewal SI. HDAC-mediated suppression of histone turnover promotes epigenetic stability of heterochromatin. Nat Struct Mol Biol. 2013;20(5):547-554.

50. Chang Y, et al. Structural basis for G9a-like protein lysine methyltransferase inhibition by BIX-01294. Nat Struct Mol Biol. 2009;16(3):312-317.

51. Coward WR, Brand OJ, Pasini A, Jenkins G, Knox AJ, Pang L. Interplay between EZH2 and G9a Regulates CXCL10 Gene Repression in Idiopathic Pulmonary Fibrosis. Am J Respir Cell Mol Biol. 2018;58(4):449-460.

52. Kattan WM, et al. Myofibroblast-Mediated Contraction. J Coll Physicians Surg Pak. 2017;27(1):38-43.

53. Shinde AV, Humeres C, Frangogiannis NG. The role of $\alpha$-smooth muscle actin in fibroblast-mediated matrix contraction and remodeling. Biochim Biophys Acta Mol Basis Dis. 2017;1863(1):298-309.

54. Milam JE, et al. PPAR-gamma agonists inhibit profibrotic phenotypes in human lung fibroblasts and bleomycin-induced pulmonary fibrosis. Am J Physiol Lung Cell Mol Physiol. 2008;294(5):L891-L901.

55. Oruqaj G, et al. Compromised peroxisomes in idiopathic pulmonary fibrosis, a vicious cycle inducing a higher fibrotic response via TGF- $\beta$ signaling. Proc Natl Acad Sci USA. 2015;112(16):E2048-E2057.

56. Rehan VK, Torday JS. PPAR $\gamma$ Signaling Mediates the Evolution, Development, Homeostasis, and Repair of the Lung. PPAR Res. 2012;2012:289867.

57. Sime PJ. The antifibrogenic potential of PPARgamma ligands in pulmonary fibrosis. J Investig Med. 2008;56(2):534-538.

58. Sassone-Corsi P. The cyclic AMP pathway. Cold Spring Harb Perspect Biol. 2012;4(12):a011148.

59. Liu X, Sun SQ, Ostrom RS. Fibrotic lung fibroblasts show blunted inhibition by cAMP due to deficient cAMP response element-binding protein phosphorylation. J Pharmacol Exp Ther. 2005;315(2):678-687.

60. Irifuku T, et al. Inhibition of $\mathrm{H} 3 \mathrm{~K} 9$ histone methyltransferase G9a attenuates renal fibrosis and retains klotho expression. Kidney Int. 2016;89(1):147-157.

61. Maeda K, et al. Inhibition of H3K9 methyltransferase G9a ameliorates methylglyoxal-induced peritoneal fibrosis. PLoS One. 2017;12(3):e0173706.

62. Shen W, Jiang XX, Li YW, He Q. Mitochondria-mediated disturbance of fatty acid metabolism in proximal tubule epithelial cells leads to renal interstitial fibrosis. Eur Rev Med Pharmacol Sci. 2018;22(3):810-819.

63. Allison SJ. Fibrosis: dysfunctional fatty acid oxidation in renal fibrosis. Nat Rev Nephrol. 2015;11(2):64.

64. Yu G, et al. Thyroid hormone inhibits lung fibrosis in mice by improving epithelial mitochondrial function. Nat Med. 2018;24(1):39-49.

65. Hagood JS. Beyond the genome: epigenetic mechanisms in lung remodeling. Physiology (Bethesda). 2014;29(3):177-185.

66. Conforti F, et al. The histone deacetylase inhibitor, romidepsin, as a potential treatment for pulmonary fibrosis. Oncotarget. 2017;8(30):48737-48754.

67. Lin J, Handschin C, Spiegelman BM. Metabolic control through the PGC-1 family of transcription coactivators. Cell Metab. 2005;1(6):361-370.

68. Baud L, Letavernier E. PPARalpha contributes to tubular protection. J Am Soc Nephrol. 2007;18(12):3017-3018.

69. Zardi EM, et al. Hepatic PPARs: their role in liver physiology, fibrosis and treatment. Curr Med Chem. 2013;20(27):3370-3396

70. Michalik L, Wahli W. Involvement of PPAR nuclear receptors in tissue injury and wound repair. J Clin Invest. 2006;116(3):598-606

71. Lakatos HF, Thatcher TH, Kottmann RM, Garcia TM, Phipps RP, Sime PJ. The Role of PPARs in Lung Fibrosis. PPAR Res. 2007;2007:71323

72. Mann J, et al. MeCP2 controls an epigenetic pathway that promotes myofibroblast transdifferentiation and fibrosis. Gastroenterology. 2010;138(2):705.

73. Tschumperlin DJ, Ligresti G, Hilscher MB, Shah VH. Mechanosensing and fibrosis. J Clin Invest. 2018;128(1):74-84.

74. Tan Y, et al. Matrix softness regulates plasticity of tumour-repopulating cells via H3K9 demethylation and Sox 2 expression. Nat Commun. 2014;5:4619.

75. Kind J, et al. Single-cell dynamics of genome-nuclear lamina interactions. Cell. 2013;153(1):178-192. 
76. van Steensel B, Belmont AS. Lamina-Associated Domains: Links with Chromosome Architecture, Heterochromatin, and Gene Repression. Cell. 2017;169(5):780-791.

77. Yata Y, et al. DNase I-hypersensitive sites enhance alpha1(I) collagen gene expression in hepatic stellate cells. Hepatology. 2003;37(2):267-276.

78. Ashcroft T, Simpson JM, Timbrell V. Simple method of estimating severity of pulmonary fibrosis on a numerical scale. J Clin Pathol. 1988;41(4):467-470. 